\title{
On the Design and Implementation of FIR and IIR Digital Filters With Variable Frequency Characteristics
}

\author{
Carson K. S. Pun, S. C. Chan, K. S. Yeung, and K. L. Ho, Member, IEEE
}

\begin{abstract}
This paper studies the design and implementation of finite-impulse response (FIR) and infinite-impulse response (IIR) variable digital filters (VDFs), whose frequency characteristics can be controlled continuously by some control or tuning parameters. A least squares (LS) approach is proposed for the design of FIR VDFs by expressing the impulse response of the filter as a linear combination of basis functions. It is shown that the optimal LS solution can be obtained by solving a system of linear equations. By choosing the basis functions as piecewise polynomials, VDFs with larger tuning range than that of ordinary polynomial based approach results. The proposed VDF can be efficiently implemented using the familiar Farrow structure. Making use of the FIR VDF so obtained, an Eigensystem Realization Algorithm (ERA)-based model reduction technique is proposed to approximate the FIR VDF by a stable IIR VDF with lower system order. The advantages of the model reduction approach are: 1) it is computational simple which only requires the computation of the singular value decomposition of a Hankel matrix; 2) the IIR VDF obtained is guaranteed to be stable; and 3 ) the frequency response such as the phase response of the FIR prototype is well preserved. Apart from the above advantages, the proposed IIR VDF does not suffer from undesirable transient response during parameter tuning found in other approaches based on direct tuning of filter parameters. For frequency selective VDFs, about $40 \%$ of the multiplications can be saved using the IIR VDFs. The implementation of the proposed FIR VDF using sum-of-powers-of-two (SOPOT) coefficient and the multiplier block (MB) technique are also studied. Results show that about two-third of the additions in implementing the multiplication of the SOPOT coefficients can be saved using the multiplier block, which leads to significant savings in hardware complexity.
\end{abstract}

Index Terms-Design and implementation, finite-impulse response (FIR) filters, infinite-impulse response (IIR) filters, least squares design, model reduction, multiplier block, variable or tunable digital filters.

\section{INTRODUCTION}

V ARIABLE digital filters (VDFs) are digital filters with controllable spectral characteristics such as variable cutoff frequency response, adjustable passband width, controllable fractional delay, etc. They find applications in different areas of signal processing and communications, e.g., fractional delay digital filters for timing adjustment in digital receivers [1],

Manuscript received April 10, 2002; revised September 16, 2002. This work was supported by the Research Grants Council of the Hong Kong Special Administrative Region of China (SAR) under Grant HKU7127/99E. This paper was recommended by Associate Editor S.-M. Phoong.

The authors are with the Department of Electrical and Electronic Engineering, The University of Hong Kong, Pokfulam Road, Hong Kong (e-mail scchan@eee.hku.hk).

Digital Object Identifier 10.1109/TCSII.2002.807574
[2]. Methods for designing variable digital filters can broadly be classified into two categories: transformation [3], [4] and spectral parameter approximation [6]-[10], [13], [14] methods. In the former, a prototype filter with certain desirable frequency characteristics is first designed. Certain transformation such as the allpass transformation method [3] is then applied to the prototype filter to obtain the final VDF. In general, transformation method is applicable to VDFs with variable cutoff frequencies, but not general variable characteristics say variable fractional delay. The spectral parameter approximation method is more general in that it assumes that either the impulse responses [7]-[10] or the poles and zeros [6], [14] of the filters are polynomials of certain spectral parameters. The coefficients of the polynomials are then determined to provide continuous tuning of the VDF by the spectral parameters. The spectral parameter method was proposed by Zarour and Fahmy [14], where the poles and zeros of an infinite impulse response (IIR) filter are assumed to be polynomials of the spectral or tuning parameters. Most of the works on VDFs reported are focused on the design of IIR VDF (see [6], [13] and references therein), and methods for guaranteeing their stability [6]. More recently, the design of 1-D [5], [10], [21] and 2-D [7], [8] finite-impulse response (FIR) VDF (by parameterizing the impulse response as polynomials) have received considerably attention due to their simple design procedure and good filter performance. Also, the close link between the Farrow-based fractional delay digital filter and such FIR VDF becomes more apparent [8].

This paper studies the design and implementation of FIR and IIR VDF. First of all, the least squares approach in [7], [10] for designing FIR VDFs is generalized to a linear combination of basis functions, which can be more general functions than polynomials. It is shown that the optimal LS solution can also be obtained by solving a system of linear equations. This differs from the weighted least squares approach in [10] in that 1) no discretization of the tuning and frequency variables are used, which helps to reduce the design complexity by means of closed form formulas (like the method proposed in [21]); 2) the approximation function is assumed to be a linear combination of basis functions. In particular, it is shown that tunable filter using a piecewise polynomial yields larger tuning range than ordinary polynomial based approach. The resulting VDF can be implemented with the familiar Farrow structure [9]. The piecewise polynomial-based approach also reduces the number of general multipliers required in the Farrow structure because of the lower 
order of the piecewise polynomial used. Making use of the FIR VDFs obtained by the proposed approach, an Eigensystem Realization Algorithm (ERA)-based model reduction technique is proposed to approximate the FIR VDF by a stable IIR VDF with lower system order. Model reduction techniques have been proposed previously to design IIR filters with approximately linear-phase. An FIR filter is first designed using the Park-McCllelan or other algorithms to meet certain specifications. Model order reduction is then applied to this FIR filter to obtain the desired IIR filter with lower system order and, hence, arithmetic complexity. The reduction process is very simple which involves the computation of the singular value decomposition of a Hankel matrix. Therefore, time consuming iterative optimization method is not necessary. In addition, the model reduced IIR system is guaranteed to be stable and it tries to preserve the frequency characteristics of the original system. If the original FIR filter is approximately linear-phase, then the reduced system will also be approximately linear-phase. Apart from the above advantages, the proposed IIR VDF does not suffer from undesirable transient response during parameter tuning found in other approaches based on direct tuning of filter parameters [6], [12], [14]-[16]. This is because the states of the IIR subfilters in the proposed structure are not abruptly changed during the parameter tuning process. Instead, their outputs are properly combined according to the tuning parameter to obtain the desired output. Since the proposed VDF structure involves a number of subfilters with fixed coefficients, it is desirable to reduce the implementation complexity of these subfilters. In the present work, the implementation of the FIR VDFs using the sum-of-powers-of-two (SOPOT) or canonical signed digits (CSD) representation of the filter coefficients and the multiplier block technique [19] is studied. More precisely, the filter coefficients of the subfilters are represented in SOPOT representation, which can be implemented with limited numbers of simple shift and addition operations. Since the tunable filter might require slightly higher order of polynomial approximation, the number of subfilters and the redundancies among their SOPOT coefficients can be considerable. To remove these redundant operations, the multiplier block (MB) method [19] is applied to the transposed form of the Farrow structure to further reduce the number of adders for its implementation. The design of the SOPOT subfilters is performed by means of a random search algorithm, which is able to determine very good candidates representing different tradeoff between arithmetic complexity and performance. Results show that about two-third of the additions in implementing the multiplication of the SOPOT coefficients can be saved using the multiplier block, which leads to significant savings in hardware complexity.

This paper is organized as follows: In Section II, the design method of the FIR VDF using the least squares method is described. The design of the IIR VDF using the ERA model reduction method is then studied in Section III. The implementation of the FIR VDF using the SOPOT representation and the multiplier block techniques is described in Section IV. Several design examples are given in Section V. Conclusions of this work are drawn in Section VI.

\section{LEASt SQuARES Design OF FIR VARIABLE Digital FILTERS}

In the spectral parameter method, the impulse response or the poles and zeros of the variable or tunable digital filters are assumed to be polynomial function of the tuning parameter. Since direct tuning of the poles and zeros will in general generate undesirable transient response during tuning, only the former will be considered. The impulse response of the variable FIR filter under consideration $h(n, \boldsymbol{\Phi})$ is assumed to be a linear combination of some functions $\psi_{m}(\boldsymbol{\Phi})$ of the spectral parameters $\boldsymbol{\Phi}$, instead of a polynomial. That is

$$
h(n, \boldsymbol{\Phi})=\sum_{m=0}^{M-1} c_{n, m} \psi_{m}(\mathbf{\Phi})
$$

where $c_{n, m}$ is the coefficient of expansion. The functions $\psi_{m}(\boldsymbol{\Phi})$ can be chosen as an orthonormal basis or other functions, depending on the applications. Our objective is to determine $c_{n, m}$ given $\psi_{m}(\boldsymbol{\Phi})$ so that the frequency response of $h(n, \boldsymbol{\Phi})$ will approximate some desirable variable frequency response as a function of $\boldsymbol{\Phi}$. First of all, let us consider the $z$-transform of the VDF as follows:

$$
H(z, \boldsymbol{\Phi})=\sum_{n=0}^{N-1} h(n, \boldsymbol{\Phi}) z^{-n}=\sum_{n=0}^{N-1} \sum_{m=0}^{M-1} c_{n, m} \psi_{m}(\mathbf{\Phi}) z^{-n} .
$$

Interchanging the order of summation, (2-2) can be rewritten as

$$
\begin{aligned}
H(z, \mathbf{\Phi}) & =\sum_{m=0}^{M-1}\left[\sum_{n=0}^{N-1} c_{n, m} z^{-n}\right] \psi_{m}(\mathbf{\Phi}) \\
& =\sum_{m=0}^{M-1} C_{m}(z) \cdot \psi_{m}(\mathbf{\Phi}) .
\end{aligned}
$$

This suggests the general structure for its implementation as shown in Fig. 1(a). It can also be viewed as a generalization of the Farrow structure for implementing a fractional delay digital filter where $h(n, \phi)$ is approximated by a polynomial in the delay parameter $\boldsymbol{\Phi}=\phi$, that is $\psi_{m}(\phi)=\phi^{m}$. If $H_{I}\left(e^{j \omega}, \boldsymbol{\Phi}\right)$ is the desired frequency response, the approximation error is

$$
E(\omega, \boldsymbol{\Phi})=H_{I}\left(e^{j \omega}, \boldsymbol{\Phi}\right)-\sum_{m=0}^{M-1} \sum_{n=0}^{N-1} c_{n, m} \psi_{m}(\boldsymbol{\Phi}) e^{-j n \omega}
$$

It can be seen that $E(\omega, \boldsymbol{\Phi})$ is a linear function of the expansion coefficients $c_{n, m}$. The $L_{2}$ norm of $E(\omega, \Phi)$ will, therefore, be a quadratic function of $c_{n, m}$, which has a unique minimum characterized by a system of linear equations. More precisely, the $L_{2}$ norm of $E(\omega, \boldsymbol{\Phi})$ is given by

$$
E=\int_{\boldsymbol{\Phi}_{S}} \int_{\Omega_{S}} W\left(e^{j \omega}, \boldsymbol{\Phi}\right) \cdot|E(\omega, \boldsymbol{\Phi})|^{2} d \omega d \boldsymbol{\Phi}
$$

where $W\left(e^{j \omega}, \boldsymbol{\Phi}\right)$ is a positive weighting function used to control the amount of approximation error in the frequency and the tuning space. The set $\Omega_{S}$ is the frequency support over which $H_{I}\left(e^{j \omega}, \boldsymbol{\Phi}\right)$ is to be approximated. For example, it can be the passband and stopband regions of a variable cutoff digital filter. 


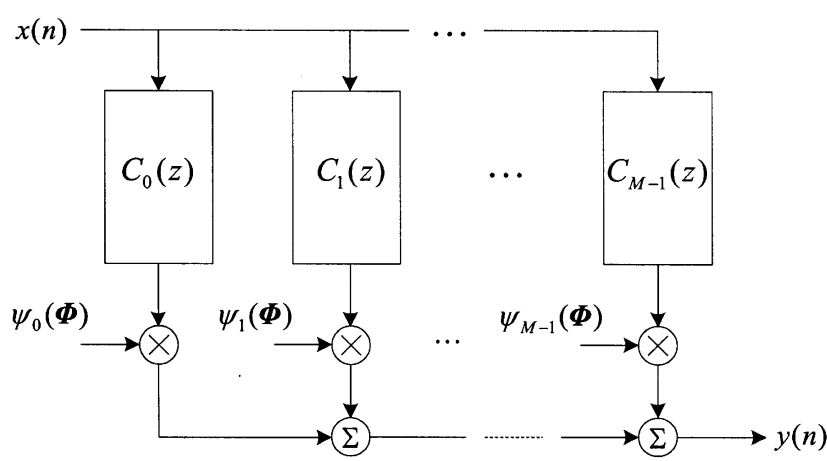

(a)

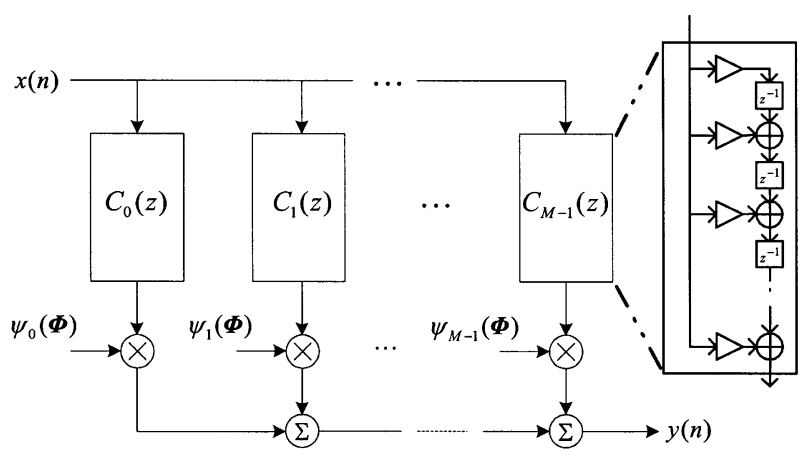

(b)

Fig. 1. (a) General FIR VDF. (b) The FIR VDF with the subfilters in transposed form.

Similarly, the set $\Phi_{S}$ is the parameter space over which the spectral parameter vector $\boldsymbol{\Phi}$ is to be varied. To simplify notation, letting $l=n+N m$ and $z=e^{j \omega}$ in (2-1), one gets

$$
\begin{aligned}
H\left(e^{j \omega}, \boldsymbol{\Phi}\right) & =\sum_{m=0}^{M-1} \sum_{n=0}^{N-1} c_{n, m} \psi_{m}(\mathbf{\Phi}) e^{-j n \omega} \\
& =\sum_{l=0}^{N M-1} a_{l} \phi_{l}(\omega, \boldsymbol{\Phi})
\end{aligned}
$$

where $a_{l}=c_{n, m}$ and $\phi_{l}(\omega, \boldsymbol{\Phi})=\psi_{m}(\boldsymbol{\Phi}) e^{-j n \omega}$. Substituting (2-6) into (2-5) and simplifying gives

$$
E=\boldsymbol{a}^{T} \boldsymbol{Q} \boldsymbol{a}-2 \boldsymbol{b}^{T} \boldsymbol{a}+c
$$

where $\quad \boldsymbol{a} \quad=\left[\begin{array}{llll}a_{0} & a_{1} & \ldots & a_{\mathrm{NM}-1}\end{array}\right]^{T}$, $\boldsymbol{b}=\left[\begin{array}{llll}b_{0} & b_{1} & \ldots & b_{N M-1}\end{array}\right]^{T}$, $[\boldsymbol{Q}]_{\mathrm{ij}}=\int_{\boldsymbol{\Phi}_{S}} \int_{\Omega_{S}} W\left(e^{j \omega}, \boldsymbol{\Phi}\right) \cdot \phi_{i}(\omega, \boldsymbol{\Phi}) \overline{\phi_{j}(\omega, \boldsymbol{\Phi}) \cdot d \omega} d \boldsymbol{\Phi}$, $[\boldsymbol{b}]_{l}=\int_{\boldsymbol{\Phi}_{S}} \int_{\Omega_{S}} W\left(e^{j \omega}, \boldsymbol{\Phi}\right) \cdot \operatorname{Re}\left\{H_{I}\left(e^{j \omega}, \boldsymbol{\Phi}\right) \overline{\phi_{l}(\omega, \boldsymbol{\Phi})}\right\} d \boldsymbol{\Phi} d \omega$, and $c=\int_{\boldsymbol{\Phi}_{S}} \int_{\Omega_{S}} W\left(e^{j \omega}, \boldsymbol{\Phi}\right) \cdot\left|H_{I}\left(e^{j \omega}, \boldsymbol{\Phi}\right)\right|^{2} d \omega d \boldsymbol{\Phi}$. Differentiating (2-7) with respect to $\boldsymbol{a}$ and setting the derivatives to zero, one gets the following system of linear equation and the optimal $L S$ solution, $\boldsymbol{a}_{L S}$ as

$$
\boldsymbol{Q} \boldsymbol{a}_{\mathrm{LS}}=\boldsymbol{b} \text { and } \boldsymbol{a}_{\mathrm{LS}}=\boldsymbol{Q}^{-1} \boldsymbol{b} \text {. }
$$

As an illustration, let us consider the design of a low-pass FIR filter with variable cutoff frequency. The passband cutoff frequencies $\omega_{p}$ and the stopband cutoff frequency $\omega_{s}$ are assumed to vary linearly with $\boldsymbol{\Phi}=\phi$ as shown in the following

$$
\begin{aligned}
& \omega_{p}(\phi)=\phi \cdot\left(\omega_{p 2}-\omega_{p 1}\right)+\omega_{p 1} \\
& \omega_{s}(\phi)=\phi \cdot\left(\omega_{s 2}-\omega_{s 1}\right)+\omega_{s 1},\left\{\Phi_{S}: \phi \in[0,1]\right\} .
\end{aligned}
$$

Therefore, the frequency support of the filter and the desired response are, respectively

$$
\begin{aligned}
& \Omega_{S}=\Omega_{p} \cup \Omega_{s}, \Omega_{p}=\left\{\omega: \omega \in\left(0, \omega_{p}(\phi)\right)\right\} \\
& \Omega_{s}=\left\{\omega: \omega \in\left(\omega_{s}(\phi), \pi\right)\right\}
\end{aligned}
$$

and

$$
H_{I}\left(e^{j \omega}, \phi\right)= \begin{cases}e^{-j \tau \omega}, & |\omega| \leq \omega_{p}(\phi) \\ 0, & \omega_{s}(\phi) \leq|\omega| \leq \pi\end{cases}
$$

where $\tau$ is the group delay which is assumed to be a constant. If $h(n, \phi)$ is approximated by a polynomial, then the function $\psi_{m}(\phi)$ is simply given by $\phi^{m}$. Putting the weighting function $W\left(e^{j \omega}, \boldsymbol{\Phi}\right)=\left\{\begin{array}{ll}K_{p}, & \omega \in S_{p} \\ K_{s}, & \omega \in S_{s}\end{array}\right.$ into (2-7), one obtains the expressions for $\boldsymbol{Q}$ and $\boldsymbol{b}$ as follows: See (2-12)-(2-13) at the bottom of the page where $i=k+N l$, and $j=n+N m$. In deriving (2-12) and (2-13), the range of integration is symmetrical about the origin. Equation (2-12) and (2-13) can readily be calculated by the reduction formula or in general numerical integration. The optimal weighted least square solution can be calculated from (2-8). The design of other variable digital filters such as variable bandpass filters and two-dimensional VDFs can be derived similarly. One problem with approximating $h(n, \phi)$ by a polynomial is that the order of the polynomial and, hence, the number of subfilters grows rapidly with the tuning range. To overcome this problem, it is desirable to approximate $h(n, \phi)$ by a piecewise polynomial in $\boldsymbol{\Phi}=\phi$. The tuning range is divided into disjoint intervals and $h(n, \phi)$ in each interval is approximated by a polynomial in $\phi$ with lower order. Fig. 2(b) shows a simple example where two piecewise polynomials with order 2 are employed. The operator $\boldsymbol{\Psi}^{-1}$ is only necessary for the IIR VDF to be discussed in Section III. For FIR VDFs, $\Psi^{-1}$ is not needed and $H_{i}(z)$ are just the subfilters $C_{m}(z)$ for the two second-order piecewise polynomials. In filtering applications where only the passband ripples, stopband attenuation and phase characteristics are of concern, the smoothing constraints between this piecewise polynomial, say continuity at the intersections of the intervals, can be relaxed. In other

$$
\begin{aligned}
{[\boldsymbol{Q}]_{i j} } & =2 \int_{0}^{1} \phi^{l+m}\left[K_{p} \sin c\left((n-k) \omega_{p}(\phi)\right)+K_{s}\left\{\sin c((n-k) \pi)-\sin c\left((n-k) \omega_{s}(\phi)\right)\right\}\right] d \phi \\
{[\boldsymbol{b}]_{j} } & =2 K_{p} \int_{0}^{1} \phi^{m} \omega_{p}(\phi) \sin c\left((n-\tau) \omega_{p}(\phi)\right) d \phi
\end{aligned}
$$




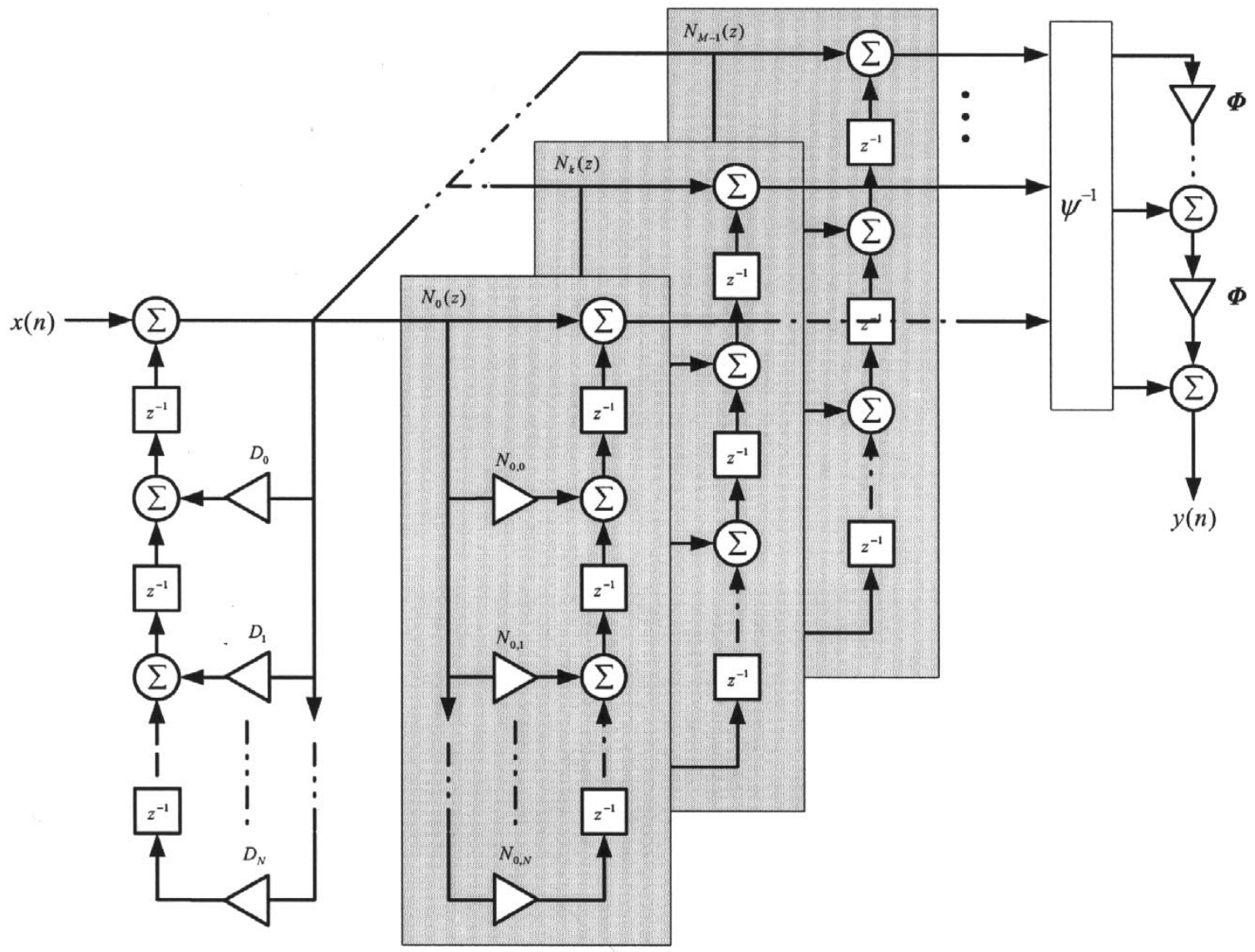

(a)

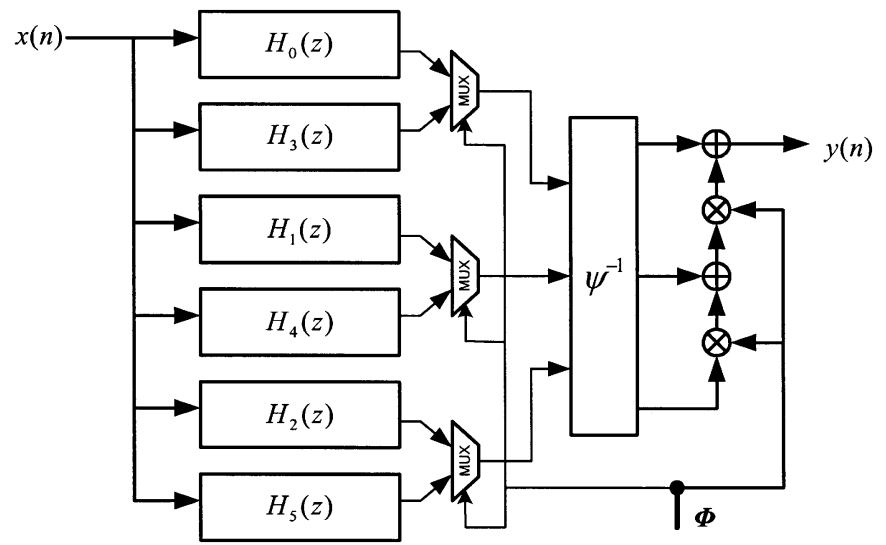

(b)

Fig. 2. (a) Proposed IIR VDF structure. (b) Proposed piecewise polynomial-based VDF structure. For FIR VDF $\Psi^{-1}=\mathbf{I}$ and $H_{i}(z)$ are the subfilters.

words, they can be designed separately using say the least squares method to meet the filter specifications. As we shall see later in Section V that this approach can considerably extend the tuning range of the VDF. In addition, the number of general multipliers to implement the VDF is also reduced. Both the proposed FIR VDF and IIR VDFs are readily generalized to two dimensions.

\section{DESIGN OF IIR VDF USING MODEL ORDER REDUCTION}

As mentioned earlier, there are several methods for designing IIR VDF. The spectral method approximates the impulse re- sponse [9], [10] of the FIR VDF or the zeros and poles of the IIR VDF [6], [14] as certain polynomials of spectral or tuning parameters. These parameters will then be used for continuous tuning of the VDF. The spectral parameter method was first proposed by Zarour and Fahmy [14], where the poles and zeros of an infinite impulse response (IIR) filter are assumed to be polynomials of the spectral or tuning parameters. Most of the works on VDFs reported focus on the design of IIR VDFs, and methods for guaranteeing their stability [6], [13]. Unlike VDFs based on FIR filters, the design of IIR VDFs requires nonlinear optimization [6], [13], [14], which is rather time consuming. Another important problem of IIR VDFs with direct 


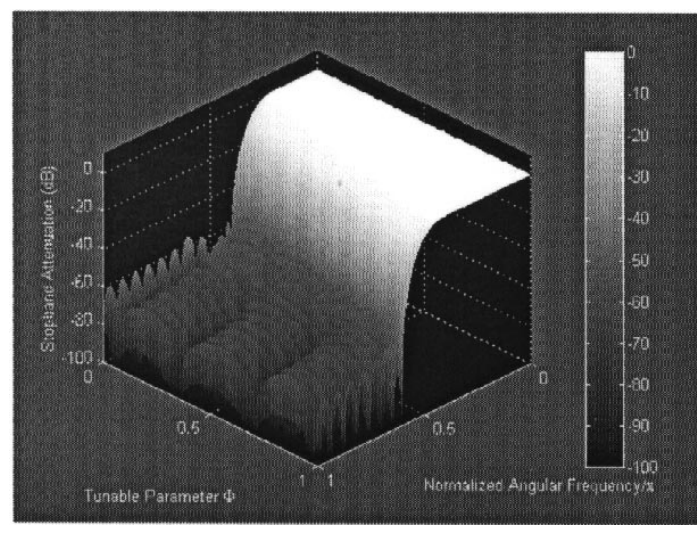

(a)

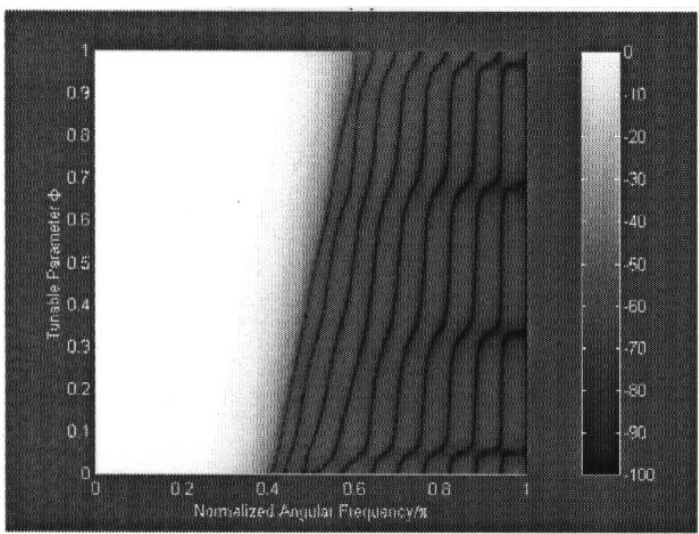

(c)

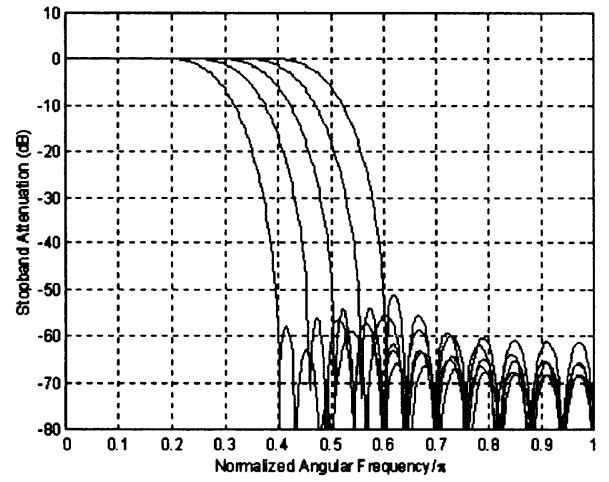

(e)

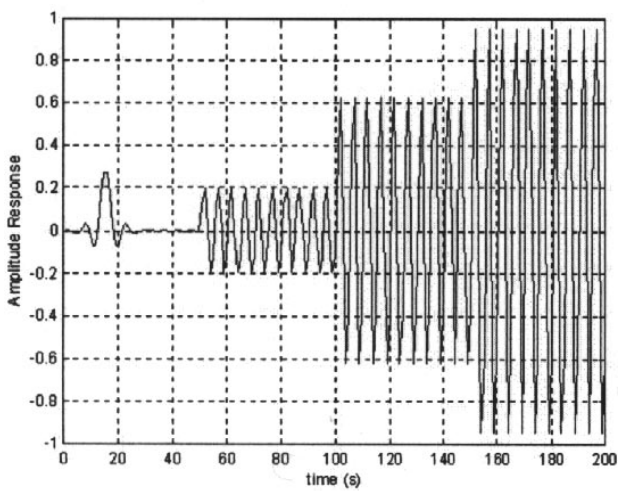

(g)

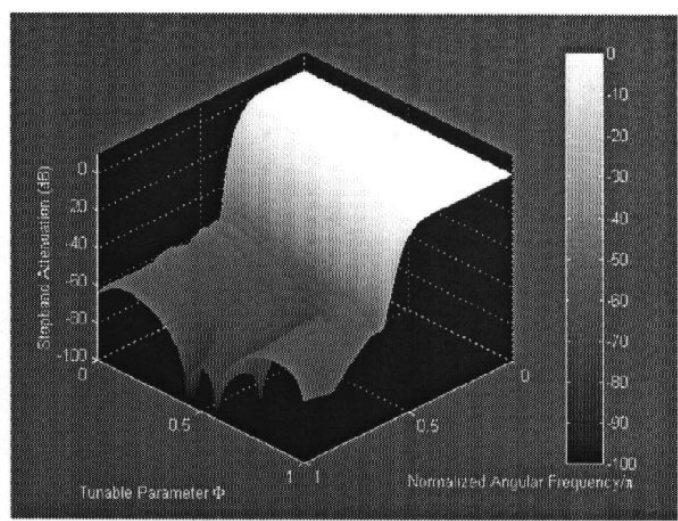

(b)

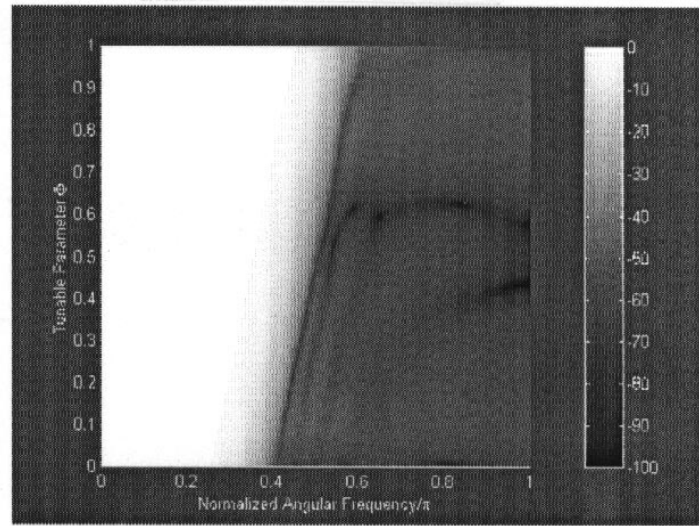

(d)

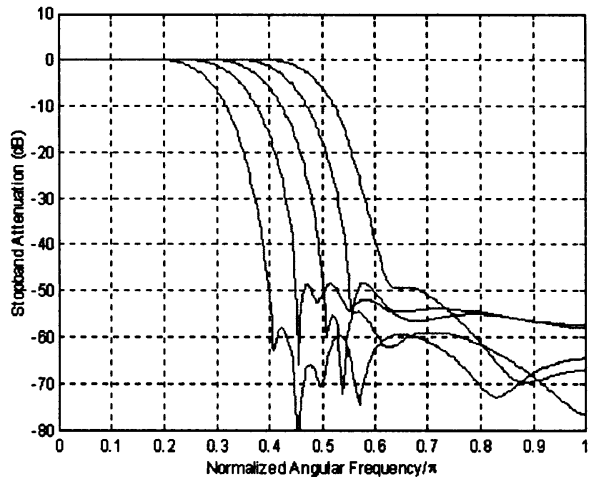

(f)

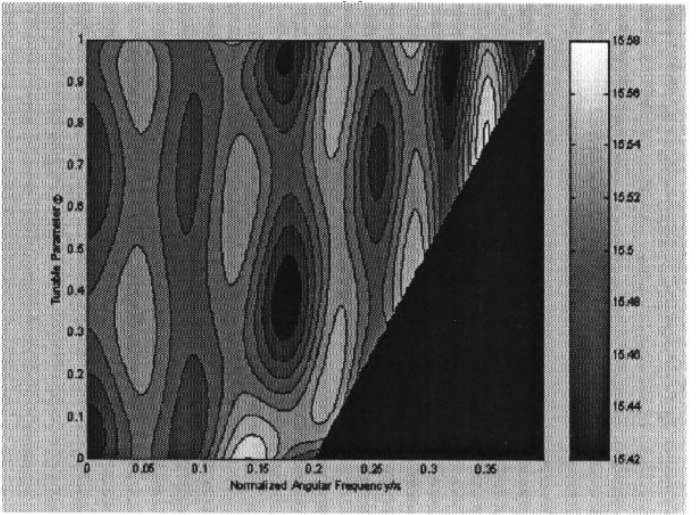

(h)

Fig. 3. Design results of Example 1. (a) and (c) Frequency responses of FIR VDF. (b) and (d) Frequency responses of IIR VDF. (e) Frequency responses of FIR VDF evenly sampled in the range $\boldsymbol{\Phi}=[0,1]$. (f) Frequency responses of IIR VDF evenly sampled in the range $\boldsymbol{\Phi}=[0,1]$. (g) Transient responses of the IIR VDF. (h) Group delay of the IIR VDF. 
tuning of the poles and zeros of the digital filters is the undesirable transient response generated during parameter tuning. It is because the previous state of the IIR filter is different from the one that is currently being used, which generates transient response with considerable magnitude. On the other hand, the VDF structure in Fig. 1 does not suffer from this problem (except at the very beginning), because the states of the subfilters are not modified during parameter tuning. Instead, their outputs are properly combined to generate the desired output. To design an IIR VDF using this structure, the subfilters $C_{m}(z)$ in (2-3) can be determined by minimizing some performance measure such as the least squares or minimax errors, subject to the filter stability constraints. This is a highly nonlinear constrained optimization problem, which is rather time consuming to solve. It might also converge to unsatisfactory local minimum, which may require repeatedly restarting the optimization procedure with different initial guess. The approach proposed in this paper is based on the model reduction of the FIR subfilters $C_{m}(z)$. Model reduction is a useful technique for designing IIR filters, especially approximately linear-phase IIR filters, from FIR filters. A FIR filter with the given specification is usually designed using the Remez or complex Remez algorithms, which are very efficient and produce optimal minimax solutions. Model reduction is then applied to convert this FIR filter to an IIR filter with similar frequency characteristic but with lower order. There are several advantages of the model reduction approach: 1) it is computational simple which only requires the computation of the singular value decomposition of a Hankel matrix; 2) the IIR VDF is guaranteed to be stable; and 3) the frequency response such as the phase response of the FIR prototype is well preserved. In other words, they can be used to design approximately linear-phase IIR filter. Direct application of model reduction to the subfilters $C_{m}(z)$, however, does not lead to satisfactory results. It is because the $C_{m}(z)$, though related to the frequency selective VDF $H(z, \boldsymbol{\Phi})$, are not frequency selective. Its coefficients are in fact the coefficients of the interpolating polynomial. Most of the singular values of the Hankel matrix of the impulse response are rather large. Model reduction, which removes the less significant singular values is, therefore, unable to offer great reduction in system order. In what follows, a transformation is used so that another set of subfilters, which is more amendable to model reduction, is implemented instead of $C_{m}(z)$. First of all, let us rewrite (2-3) in matrix form as follows:

$$
H(z, \boldsymbol{\Phi})=\boldsymbol{\Gamma}^{T} \boldsymbol{P}(\boldsymbol{\Phi})
$$

where $\boldsymbol{\Gamma}=\left[\begin{array}{lll}C_{0}(z) & \ldots & C_{M-1}(z)\end{array}\right]^{T}$ and $\boldsymbol{P}(\boldsymbol{\Phi})=$ $\left[\begin{array}{llll}\psi_{0}(\boldsymbol{\Phi}) & \ldots & \psi_{M-1}(\boldsymbol{\Phi})\end{array}\right]^{T}$. Sampling the transfer function $H(z, \boldsymbol{\Phi})$ at $M$ values of the tuning parameter $\boldsymbol{\Phi}=\boldsymbol{\Phi}_{i}, i=0, \ldots M-1$, yields

$$
H\left(z, \boldsymbol{\Phi}_{i}\right)=\boldsymbol{\Gamma}^{T} \boldsymbol{P}\left(\boldsymbol{\Phi}_{i}\right)=\boldsymbol{\Gamma}^{T} \boldsymbol{P}_{i}
$$

where $\boldsymbol{P}_{i}=\left.\boldsymbol{P}(\boldsymbol{\Phi})\right|_{\boldsymbol{\Phi}=\boldsymbol{\Phi}_{i}}$. Equation (3-2) can also be written in matrix form as follows:

$$
\boldsymbol{H}=\boldsymbol{\Psi}^{T} \cdot \boldsymbol{\Gamma}
$$

TABLE I

PARAMETERS FOR THE TUNABLE LOWPASS FILTER IN EXAMPLE $\boldsymbol{I}$

\begin{tabular}{l|c|c}
\hline & FIR VDF & IIR VDF \\
\hline Filter length & 32 & $\begin{array}{c}17 \text { (numerator) } \\
17 \text { (denominator) }\end{array}$ \\
\hline $\begin{array}{l}\text { Interpolation order } \\
\begin{array}{l}\text { Total Number of Multiplications (due } \\
\text { to filter coefficients) }\end{array}\end{array}$ & $32 \times 6=192$ & 5 \\
\hline $\begin{array}{l}\text { Total Number of Delays (due to the } \\
\text { tapped delay line) }\end{array}$ & $31 \times 6=186$ & $17 \times(6+1)=119$ \\
\hline $\begin{array}{l}\text { Total Number of Additions (due to the } \\
\text { tapped delay line) }\end{array}$ & $31 \times 6=186$ & $16 \times(6+1)=112$ \\
\hline Passband Ripple & $0.00527(0.056 \mathrm{~dB})$ & $0.00717(0.062 \mathrm{~dB})$ \\
\hline Stopband Ripple & $0.00645(43.80 \mathrm{~dB})$ & $0.01218(38.29 \mathrm{~dB})$ \\
\hline
\end{tabular}

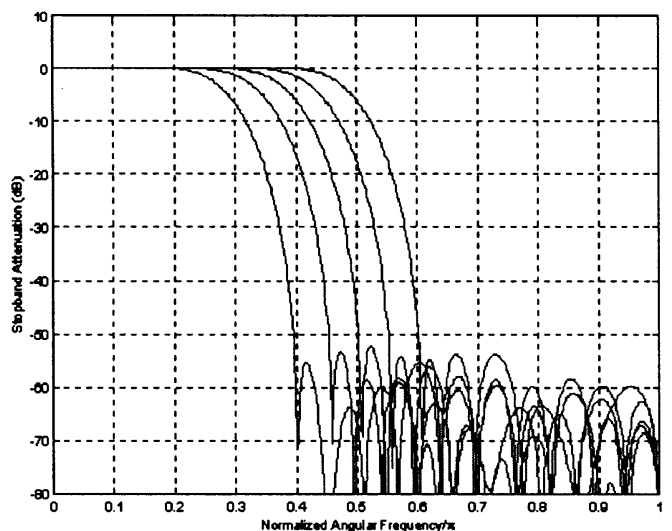

(a)

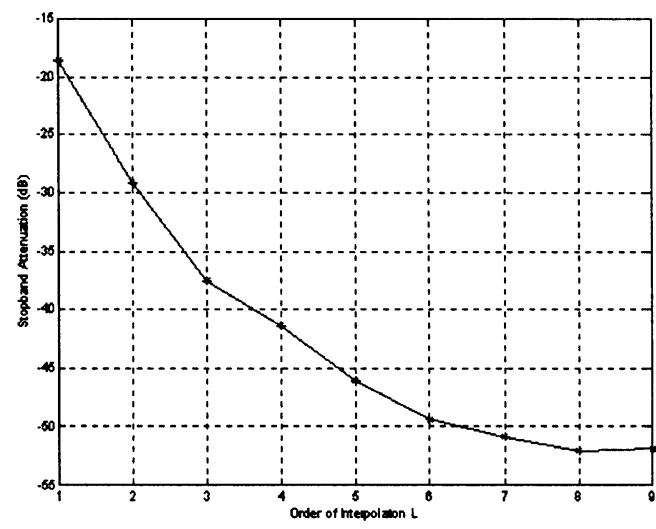

(b)

Fig. 4. (a) Frequency responses of multiplier-less FIR VDF evenly sampled in the range $\boldsymbol{\Phi}=[0,1]$. (b) Worst-case stopband attenuation as a function of the order of the interpolation polynomial.

TABLE II

PARAMETERS FOR THE EFFICIENT IMPLEMENTATION OF THE TUNABLE LOWPASS FILTER IN EXAMPLE 1

\begin{tabular}{l|c}
\hline \multicolumn{1}{c|}{ Interpolation order } & 5 \\
\hline Filter order & 31 \\
\hline Cutoff frequency tuning range & $0.2 \pi-0.4 \pi$ \\
\hline Transition bandwidth & $0.2 \pi$ \\
\hline Worst-case stopband attenuation & $46.1 \mathrm{~dB}$ \\
\hline Average no. of terms of SOPOT coefficient & 4.1 \\
\hline $\begin{array}{l}\text { Required adders before using MB } \\
\text { (including adders in the delay chain) }\end{array}$ & $822=6 \times 31+636$ \\
\hline $\begin{array}{l}\text { Required adders after using MB } \\
\text { (including adders in the delay chain) }\end{array}$ & $271=6 \times 31+85$ \\
\hline
\end{tabular}




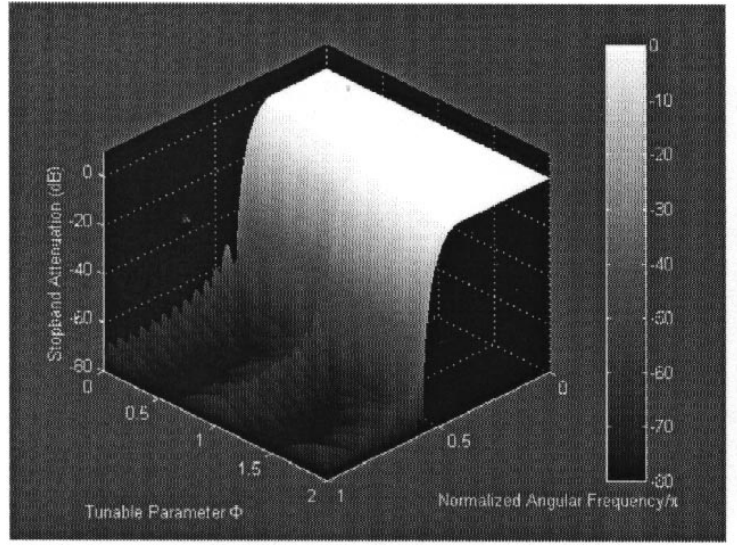

(a)

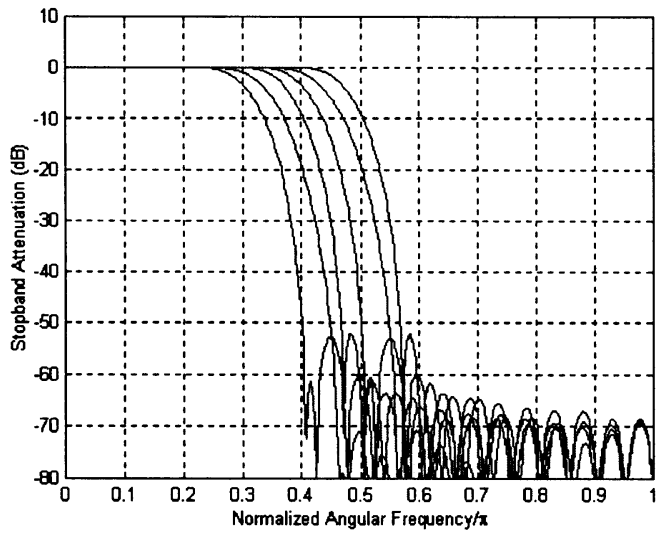

(c)

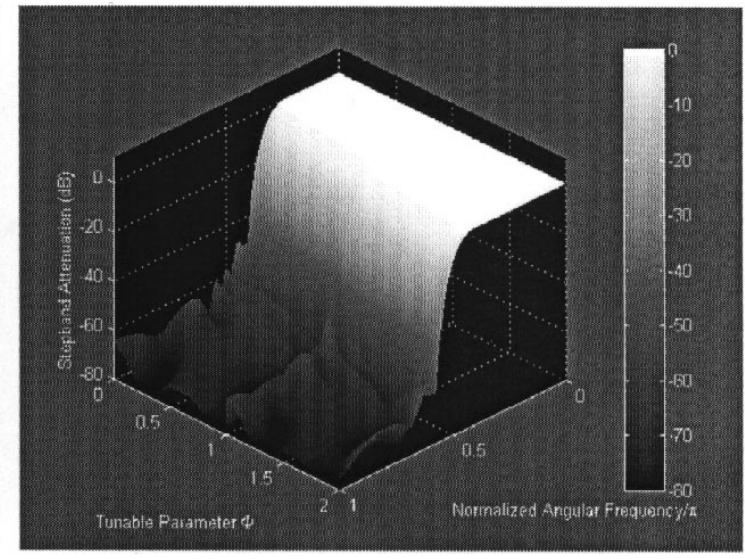

(b)

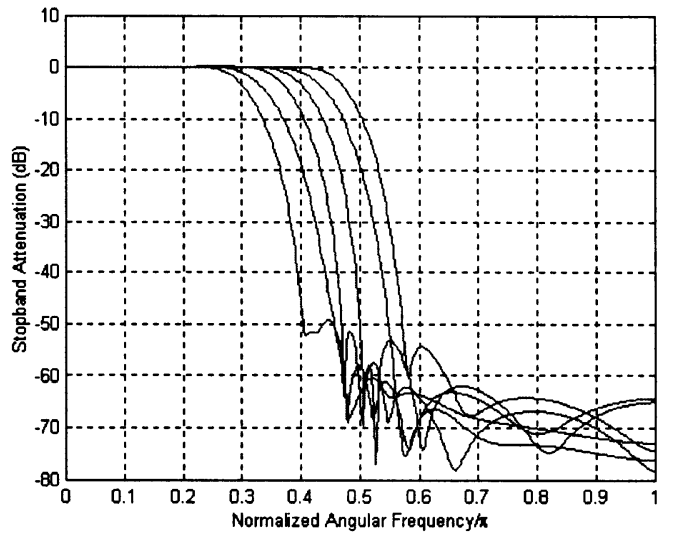

(d)

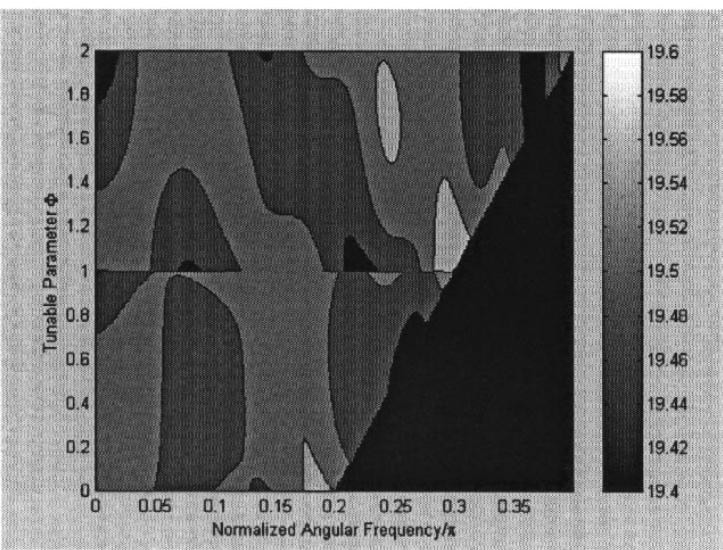

(e)

Fig. 5. Design results of Example 2. (a) Frequency responses of FIR VDF. (b) Frequency responses of IIR VDF. (c) Frequency responses of FIR VDF evenly sampled in the range $\boldsymbol{\Phi}=[0,2]$. (d) Frequency responses of IIR VDF evenly sampled in the range $\boldsymbol{\Phi}=[0,2]$. (e) Group delay of the IIR VDF.

where $\boldsymbol{\Psi}=\left[\begin{array}{lll}\mathbf{P}_{0} & \ldots & \mathbf{P}_{M-1}\end{array}\right]^{T}$ is a $M \times M$ matrix and $\boldsymbol{H}=\left[\begin{array}{lll}H\left(z, \mathbf{\Phi}_{0}\right) & \ldots & H\left(z, \boldsymbol{\Phi}_{M-1}\right)\end{array}\right]^{T}$. If $\boldsymbol{\Psi}$ in (3-3) is nonsingular, then we can express $\boldsymbol{\Gamma}$ in terms of $\boldsymbol{H}$ as follows:

$$
\boldsymbol{\Gamma}=\boldsymbol{\Psi}^{-1} \cdot \boldsymbol{H} .
$$

In other words, the subfilters $C_{m}(z)$ 's can be replaced by another set of subfilters $H\left(z, \boldsymbol{\Phi}_{i}\right)$ followed by a linear transformation $\boldsymbol{\Psi}^{-1}$. For polynomial basis functions, we have $\psi_{m}(\boldsymbol{\Phi})=\phi^{m}$. If the $M$ values of $\boldsymbol{\Phi}$ are evenly spaced, i.e., $\boldsymbol{\Phi}_{i}=\phi_{i}$ is chosen as $(i / M)$, then $\boldsymbol{\Psi}$ is the Vandermonde matrix and it is nonsingular. The advantage of this transformation, as mentioned earlier, is that model reduction will be applied to $H\left(z, \boldsymbol{\Phi}_{i}\right)$, which will produce a reduced system with lower order than using $C_{m}(z)$, if $H(z, \boldsymbol{\Phi})$ is frequency selective. For example, if $H(z, \boldsymbol{\Phi})$ is a low-pass filter with variable cutoff frequency, $H\left(z, \boldsymbol{\Phi}_{i}\right)$ will be the desired low-pass filter with cutoff frequency governed by $\boldsymbol{\Phi}_{i}$. When $H\left(z, \boldsymbol{\Phi}_{i}\right)$ undergoes model order reduction, it will yield much lower order than that from model reducing $C_{m}(z)$, which is no longer a low-pass filter. It is because $H\left(z, \boldsymbol{\Phi}_{i}\right)$ 's are frequency selective and are similar in frequency characteristic. They are thus more amendable to model reduction. 
As mentioned earlier, designing VDF with wide tuning range using the polynomial basis will in general require large number of branches. This will increase the order of the matrix $\Psi^{-1}$ and, hence, the complexity. To remedy this problem, it is desirable to split the tuning range into several consecutive tuning ranges. A separate FIR VDF is designed for each segment, which helps to reduce the order of the interpolation polynomial and, hence, the number of branches. In addition, the number of general multiplier required and the order of the matrix $\Psi^{-1}$ will also be reduced. Next we consider the model order reduction of $H\left(z, \boldsymbol{\Phi}_{i}\right)$. The algorithm that we employed is the Eigensystem realization algorithm (ERA) [17], [18]. First of all, we note that the subfilters $C_{m}(z)$ can be viewed as a single input-multiple output (SIMO) system. To carry out model reduction of these subfilters, we first rewrite them in state space model (SSM) with order $N-1$ [22], where $N$ is the filter length of $H\left(z, \boldsymbol{\Phi}_{i}\right)$ in (3-2), as follows:

$$
\begin{aligned}
\boldsymbol{x}(k+1) & =\boldsymbol{A} \cdot \boldsymbol{x}(k)+\boldsymbol{B} u(k) \\
\boldsymbol{y}(k) & =\boldsymbol{C} \cdot \boldsymbol{x}(k)+\boldsymbol{D} \cdot u(k)
\end{aligned}
$$

where

$$
\begin{aligned}
\boldsymbol{A} & =\left[\begin{array}{cc}
\boldsymbol{O}_{\boldsymbol{N}-\mathbf{2}}^{\boldsymbol{T}} & 0 \\
\boldsymbol{I}_{\boldsymbol{N - 2}} & \boldsymbol{O}_{\boldsymbol{N - 2}}
\end{array}\right], \quad \boldsymbol{B}=\left[\begin{array}{c}
1 \\
\boldsymbol{O}_{\boldsymbol{N}-\mathbf{2}}
\end{array}\right], \\
\boldsymbol{C} & =\left[\begin{array}{ccc}
c_{1,0} & \ldots & c_{N-1,0} \\
\vdots & \ddots & \vdots \\
c_{1, M-1} & \ldots & c_{N-1, M-1}
\end{array}\right], \quad \boldsymbol{D}=\left[\begin{array}{c}
c_{0,0} \\
\vdots \\
c_{0, M-1}
\end{array}\right]
\end{aligned}
$$

$\boldsymbol{O}_{\boldsymbol{N}}$ is a $N \times 1$ zero matrix, and $\boldsymbol{I}_{\boldsymbol{N}}$ is an $N \times N$ identity matrix. Let $\boldsymbol{Y}_{k}, k=1,2, \ldots$, be the $(M \times 1)$ pulse-response matrix or Markov parameters obtained by applying a single impulse to the input at $k=0$, i.e., $u(0)=1$ and $u(k)=0(k=1,2, \ldots)$. From the state space model in (3-5) and (3-6), one gets the following formula for the Markov parameters:

$Y_{0}=D, Y_{1}=C B, Y_{2}=C A B, \ldots, Y_{k}=C A^{k-1} B, \ldots$

The ERA system begins by forming the generalized $\alpha M \times \beta$ Hankel matrix $H(k-1)$ composed of the Markov parameters as follows:

$$
\boldsymbol{H}(k-1)=\left[\begin{array}{cccc}
\boldsymbol{Y}_{\boldsymbol{k}} & \boldsymbol{Y}_{\boldsymbol{k}+\mathbf{1}} & \ldots & \boldsymbol{Y}_{\boldsymbol{k}+\boldsymbol{\beta}-\mathbf{1}} \\
\boldsymbol{Y}_{\boldsymbol{k}+\mathbf{1}} & \boldsymbol{Y}_{\boldsymbol{k}+2} & \ldots & \boldsymbol{Y}_{\boldsymbol{k}+\boldsymbol{\beta}} \\
\vdots & \vdots & \ddots & \vdots \\
\boldsymbol{Y}_{\boldsymbol{k}+\boldsymbol{\alpha}-1} & \boldsymbol{Y}_{\boldsymbol{k}+\boldsymbol{\alpha}} & \ldots & \boldsymbol{Y}_{\boldsymbol{k}+\boldsymbol{\alpha}+\boldsymbol{\beta}-2}
\end{array}\right]
$$

For simplicity, we choose $\alpha=\beta=N-1$ (order of SSM), and $k=1$. Next, the singular value decomposition (SVD) of the Hankel matrix with $k=1$ is computed

$$
\boldsymbol{H}(0)=\boldsymbol{R} \cdot \sum \cdot \boldsymbol{S}^{T}
$$

TABLE III

PARAMETERS FOR THE TUNABle LOWPASS FILTERS IN EXAMPLE 2 [(TwO BlOCKS EACH WITH THREE BRANCHES. EACH BLOCK USES LaGRANGE INTERPOLATOR (ORDER-TWO)]

\begin{tabular}{l|c|c}
\hline & FIR VDF & IIR VDF \\
\hline Filter length & 40 & $\begin{array}{c}21 \text { (numerator) } \\
21 \text { (denominator) }\end{array}$ \\
\hline *Interpolation order & 2 & 2 \\
\hline $\begin{array}{l}\text { Total Number of Multiplications (due to } \\
\text { filter coefficients) }\end{array}$ & $40 \times 6=240$ & $21 \times(6+1)=147$ \\
$\begin{array}{l}\text { Total Number of Delays (due to the } \\
\text { tapped delay line) }\end{array}$ & $39 \times 6=234$ & $20 \times(6+1)=140$ \\
\hline $\begin{array}{l}\text { Total Number of Additions (due to the } \\
\text { tapped delay line) }\end{array}$ & $39 \times 6=234$ & $20 \times(6+1)=140$ \\
\hline Passband Ripple & $0.00258(0.022 \mathrm{~dB})$ & $0.01594(0.1374 \mathrm{~dB})$ \\
\hline Stopband Ripple & $0.00541(45.34 \mathrm{~dB})$ & $0.00609(44.31 \mathrm{~dB})$ \\
\hline
\end{tabular}

TABLE IV

PARAMETERS FOR THE EFFICIENT IMPLEMENTATION OF THE TUNABLE LOWPASS FILTER IN EXAMPLE 2

\begin{tabular}{l|c}
\hline Interpolation order & 2 \\
\hline Number of intervals & 2 \\
\hline Filter order & 39 \\
\hline Cutoff frequency tuning range & $0.2 \pi-0.4 \pi$ \\
\hline Transition bandwidth & $0.2 \pi$ \\
\hline Worst-case stopband attenuation & $50 \mathrm{~dB}$ \\
\hline Average number of terms of SOPOT coefficient & 3.8 \\
\hline Required adders before using MB $*$ & $952=6 \times 39+718$ \\
\hline Required adders after using MB $*$ & $337=6 \times 39+103$ \\
\hline
\end{tabular}

*: include the adders in the delay chain.

where the columns of matrices $\boldsymbol{R}$ and $\boldsymbol{S}$ are orthonormal and $\Sigma$ is a rectangular matrix given by

$$
\begin{aligned}
\sum= & {\left[\begin{array}{ll}
\sum_{n} & 0 \\
0 & 0
\end{array}\right] } \\
& \text { with } \sum_{n}=\operatorname{diag}\left[\sigma_{1}, \sigma_{2}, \ldots, \sigma_{i}, \sigma_{i+1}, \ldots, \sigma_{n}\right]
\end{aligned}
$$

$\sigma_{i}(i=1,2, \ldots, n)$, are the singular values arranged in descending order of their magnitude

$$
\sigma_{1} \geq \sigma_{2} \geq \cdots \geq \sigma_{i} \geq \sigma_{i+1} \geq \cdots \geq \sigma_{n} \geq 0
$$

Let $r$ be the order of the model-reduced system and $\boldsymbol{R}_{r}$ and $\boldsymbol{S}_{r}$ be the matrices formed by the first $r$ columns of $\boldsymbol{R}$ and $\boldsymbol{S}$, respectively. Similar, let $\Sigma_{r}$ be the matrix formed by the first $r$ columns and first $r$ rows of $\boldsymbol{\Sigma}$. To reduce the $(\boldsymbol{A}, \boldsymbol{B}, \boldsymbol{C}, \boldsymbol{D})$ matrices to the reduced system $\left(\boldsymbol{A}_{r}, \boldsymbol{B}_{r}, \boldsymbol{C}_{r}, \boldsymbol{D}_{r}\right)$, let us form the following reduced Hankel matrix:

$$
\boldsymbol{H}_{r}(0)=\boldsymbol{R}_{r} \sum_{r} \boldsymbol{S}_{r}^{T}
$$

It can be shown that $\boldsymbol{H}_{r}(0)$ is composed of the controllability matrix $\boldsymbol{M}_{C}$ and the observability matrix $\boldsymbol{M}_{o}$ as follows:

$$
\boldsymbol{H}_{r}(0)=\boldsymbol{M}_{c} \cdot \boldsymbol{M}_{o}
$$

where $\boldsymbol{M}_{c}=\left[\begin{array}{llll}\boldsymbol{C}_{r} & \boldsymbol{C}_{r} \boldsymbol{A}_{r} & \cdots & \boldsymbol{C}_{r} \boldsymbol{A}_{r}^{\alpha-1}\end{array}\right]^{T}, \boldsymbol{M}_{o}=$ $\left[\begin{array}{llll}\boldsymbol{B}_{r} & \boldsymbol{A}_{r} \boldsymbol{B}_{r} & \cdots & \boldsymbol{A}_{r}^{\beta-1} \boldsymbol{B}_{r}\end{array}\right]$. More generally, we have

$$
\boldsymbol{H}_{r}(k)=\boldsymbol{M}_{c} \boldsymbol{A}_{r}^{k} \boldsymbol{M}_{o} .
$$




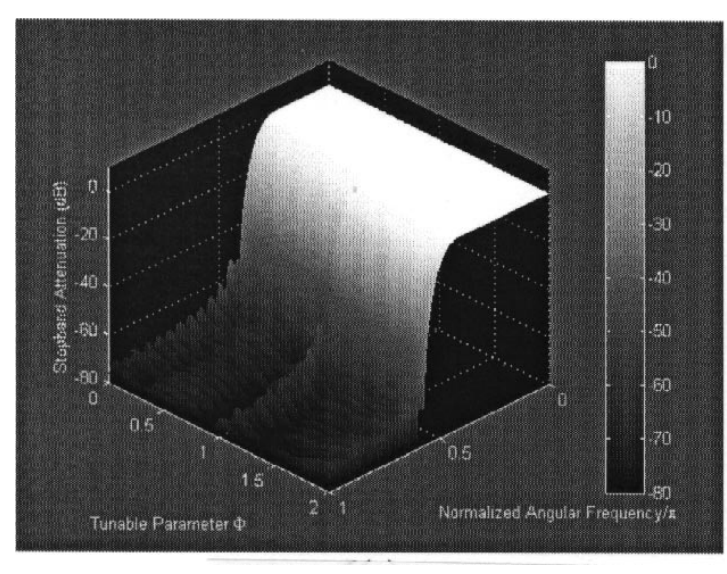

(a)

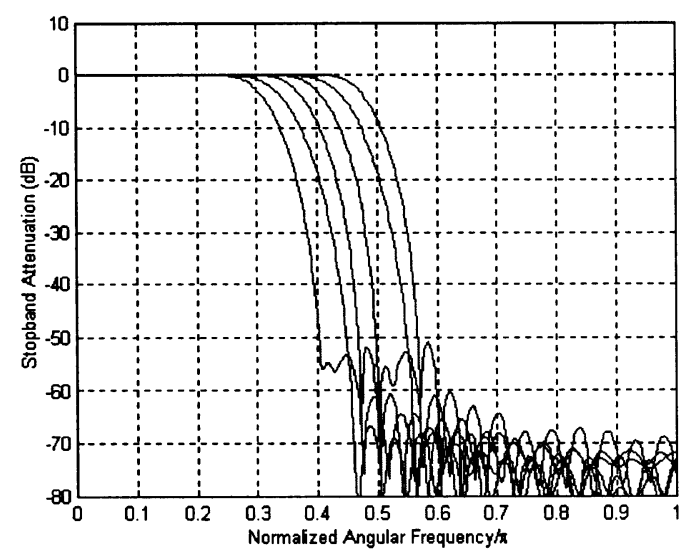

(c)

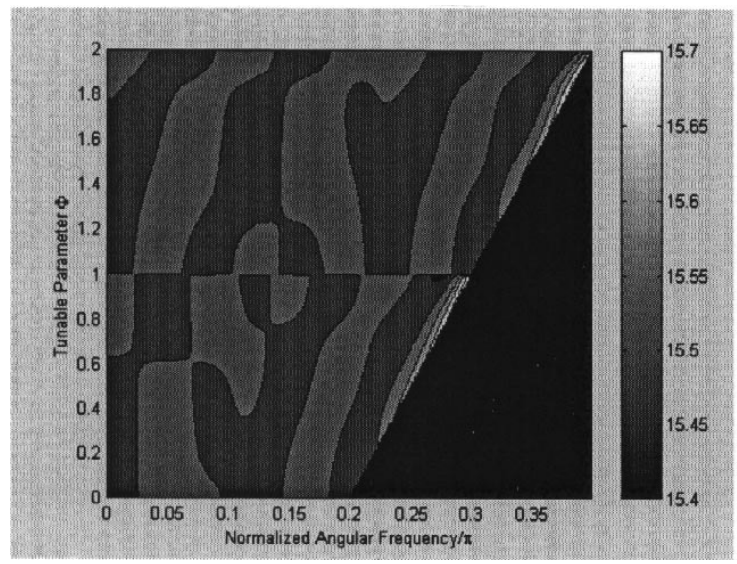

(e)

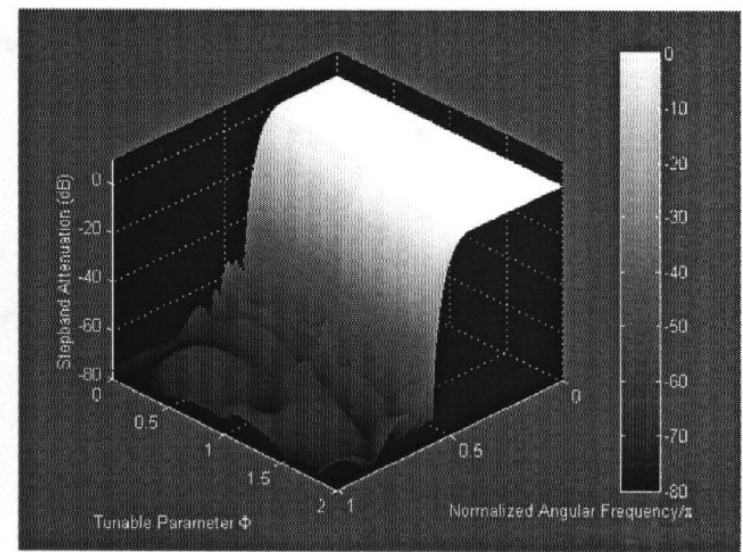

(b)

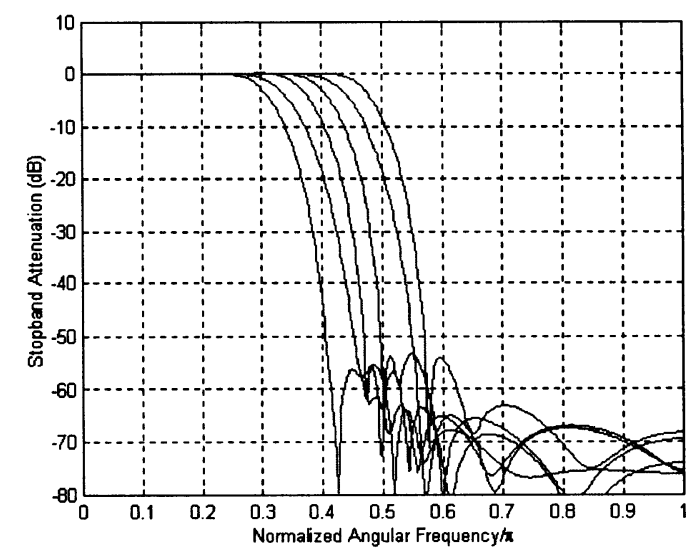

(d)

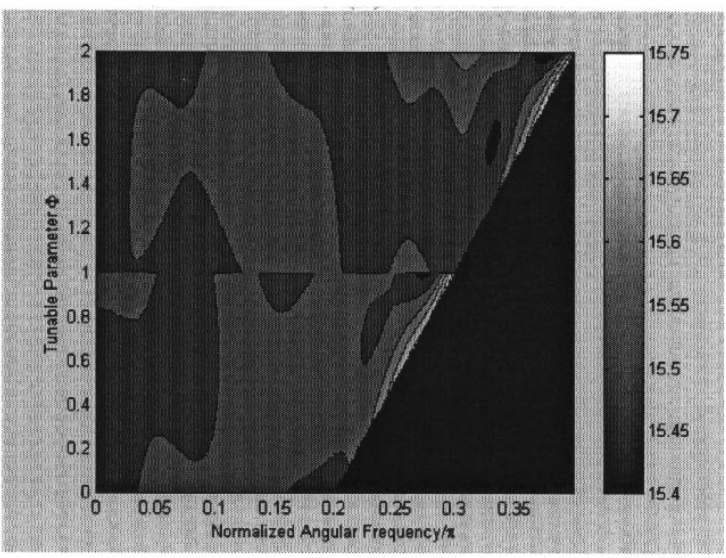

(f)

Fig. 6. Design results in Example 2. (a) Frequency response of FIR VDF. (b) Frequency response of IIR VDF. (c) Frequency response of FIR VDF evenly sampled in the range $\boldsymbol{\Phi}=[0,2]$. (d) Frequency response of IIR VDF evenly sampled in the range $\boldsymbol{\Phi}=[0,2]$. (e) Group delay of the FIR VDF. (f) Group delay of the IIR VDF.

Comparing (3-12) and (3-13) with $k=0$ gives

$$
\boldsymbol{M}_{c}=\boldsymbol{R}_{r} \sum_{r}^{1 / 2}, \text { and } \boldsymbol{M}_{o}=\sum_{r}^{1 / 2} \boldsymbol{S}_{r}^{T}
$$

From (3-13), it is clear that the first column of $M_{o}$ forms the reduced input matrix $\boldsymbol{B}_{r}$ whereas the first $M$ rows of $\boldsymbol{M}_{c}$ form the reduced output matrix $\boldsymbol{C}_{r}$. The reduced $\boldsymbol{D}_{r}$ matrix is exactly equal to the $\boldsymbol{D}$ matrix. To determine $\boldsymbol{A}_{r}$, consider (3-14) and (3-15) with $k=1$

$$
\boldsymbol{H}_{\boldsymbol{r}}(1)=\boldsymbol{M}_{\boldsymbol{C}} \boldsymbol{A}_{\boldsymbol{r}} \boldsymbol{M}_{\boldsymbol{O}}=\boldsymbol{R} \boldsymbol{r} \sum_{r}^{1 / 2} A_{\boldsymbol{r}} \sum_{r}^{1 / 2} \boldsymbol{S}_{r}^{T} .
$$

Rearranging, one gets the following reduced state matrix $A_{r}$

$$
\boldsymbol{A}_{r}=\sum_{r}^{-1 / 2} \boldsymbol{R}_{r}^{T} \boldsymbol{H}_{r}(1) \boldsymbol{S}_{r} \sum_{r}^{-1 / 2} .
$$


Now, all $\left(\boldsymbol{A}_{r}, \boldsymbol{B}_{r}, \boldsymbol{C}_{r}, \boldsymbol{D}_{r}\right)$ matrices have been determined. Using the reduced state space model, one can determine a new set of transfer functions, which have a common denominator. Thus, the system transfer function will consist of $M$ numerators and a denominator, all with order $r$. An important property of the ERA algorithm is that the reduced system is stable if the original system is stable. Moreover, since model reduction will try to preserve the frequency characteristics of the original system, the IIR filter obtained will be approximately linear-phase, if the original FIR VDF is linear or approximately linear-phase.

Let the model-reduced vector of $\boldsymbol{H}$ be denoted as $\tilde{\boldsymbol{H}}$. As mentioned earlier, the new reduced transfer function will have the same denominator $D(z)$ and the $M$ numerators, denoted by $N_{i}(z)$. Therefore, we have

$$
\tilde{\boldsymbol{H}}=\frac{\boldsymbol{N}}{D(z)}
$$

where $\boldsymbol{N}=\left[\begin{array}{lll}N_{0}(z) & \cdots & N_{M-1}(z)\end{array}\right]^{T}$. The final SIMO model-reduced transfer function is

$$
\boldsymbol{\Gamma}=\boldsymbol{\Psi}^{-1} \cdot \frac{\boldsymbol{N}^{T}}{D(z)} .
$$

Since there is only one denominator, the implementation complexity associated with the denominator of the transfer functions is greatly reduced. In addition, for certain types of tunable filter, the use of SIMO ERA can dramatically reduce the order of the system and, hence, the arithmetic complexity, as we will see later in the design examples. The structure of the final IIR VDF is shown in Fig. 2(a).

The design method of the proposed IIR VDF is summarized as follows.

1) Design an FIR VDF according to the design specification using the polynomial basis or piecewise polynomial basis. This gives the subfilters $\Gamma$.

2) Using $\boldsymbol{\Gamma}$, compute the transfer function $H(z, \boldsymbol{\Phi})$ at evenly sampled values of $\boldsymbol{\Phi}, H\left(z, \boldsymbol{\Phi}_{i}\right)=\boldsymbol{\Gamma}^{T} \boldsymbol{P}\left(\boldsymbol{\Phi}_{i}\right)$. This gives $\boldsymbol{H}=\boldsymbol{\Psi}^{T} \cdot \boldsymbol{\Gamma}$.

3) Apply the ERA model reduction method to the SIMO system $\boldsymbol{H}$. This gives $\tilde{\boldsymbol{H}}=\boldsymbol{N} / D(z)$.

Finally, it is noted that the implementation of $\boldsymbol{\Psi}^{-1}$ can be avoided by forming $\hat{\boldsymbol{N}}=\boldsymbol{\Psi}^{-1} \cdot \boldsymbol{N}^{T}$ offline. The efficient implementation of the FIR VDF will be considered in the following section. Due to space limitation, the hardware implementation of the IIR VDF is not discussed in this paper.

\section{EFFICIENT IMPLEMENTATION OF FIR VDF}

To reduce the implementation complexity, the subfilters are implemented as multiplier-less FIR filters using the SOPOT coefficients in the form

$$
\hat{c}_{n, m}=\sum_{j=1}^{L_{s}} b_{n, m, j} \cdot 2^{a_{j}}
$$

where $b_{n, m, j} \in\{-1,1\}$ and $a_{j} \in\{-l, \ldots,-1,0,1, \ldots, l\} . l$ is a positive integer and its value determines the range of the
TABLE V

PARAMETERS FOR THE TUNABLE LOW-DELAY LOWPASS FILTERS IN EXAMPLE 2 (Two Blocks, Each With ThreE BRanches. Each Block Uses LAGRANGE INTERPOLATOR OF ORDER-TWO)

\begin{tabular}{l|c|c}
\hline & FIR VDF & IIR VDF \\
\hline Filter length & 40 & $\begin{array}{c}21 \text { (numerator) } \\
21 \text { (denominator) }\end{array}$ \\
\hline${ }^{*}$ Interpolation order & 2 & 2 \\
\hline $\begin{array}{l}\text { Total Number of Multiplications (due to } \\
\text { filter coefficients) }\end{array}$ & $40 \times 6=240$ & $21 \times(6+1)=147$ \\
\hline $\begin{array}{l}\text { Total Number of Delays (due to the } \\
\text { tapped delay line) }\end{array}$ & $39 \times 6=234$ & $20 \times(6+1)=140$ \\
\hline $\begin{array}{l}\text { Total Number of Additions (due to the } \\
\text { tapped delay line) }\end{array}$ & $39 \times 6=234$ & $20 \times(6+1)=140$ \\
\hline Passband Ripple & $0.00177(0.0154 \mathrm{~dB})$ & $0.01424(0.1228 \mathrm{~dB})$ \\
\hline Stopband Ripple & $0.00633(43.97 \mathrm{~dB})$ & $0.00781(42.15 \mathrm{~dB})$ \\
\hline
\end{tabular}

coefficients, and $L_{s}$ is the number of terms used in the coefficient approximation and is usually limited to a small number. The coefficient multiplication can then be implemented as limited number of shifts and additions. To design the SOPOT subfilters, we minimize the $L_{\infty}$ norm of its difference in frequency response with the ideal one as shown in the following:

$$
\delta_{a}=\max _{\omega \in \Omega_{s}, \mathbf{\Phi} \in[0,1]}\left\langle\left|H_{I}\left(e^{j \omega}\right)-\hat{H}\left(e^{j \omega}\right)\right|\right\rangle
$$

$H_{I}\left(e^{j \omega}\right)$ is the ideal frequency response and $\hat{H}\left(e^{j \omega}\right)$ is the frequency response calculated for a given SOPOT filter coefficients. In other words, we try to minimize the peak ripple error $\delta_{a}$ for the whole frequency range of interest and the whole tunable range $\Phi \in[0,1]$. The design procedure consists of two stages. First, the filter coefficients $b_{n, m, j}$ are optimized using a random search algorithm with respect to the criteria stated in (4-2). Then, the technique of the multiplier block is used to further reduce the number of adders required to implement all the SOPOT coefficients.

To be more specific, the real-valued coefficients $c_{n, m}$ are first determined by the least square method described in Section II. Let $\boldsymbol{b}$ be the vector containing these coefficients. Then the random search algorithm will repetitively calculate a candidate SOPOT vector $\boldsymbol{b}_{c}$ by adding to $\boldsymbol{b}$ a random perturbation vector $\lambda \boldsymbol{b}_{p}$ and then rounding it to the nearest SOPOT representation. That is

$$
\boldsymbol{b}_{c}=\left\lfloor\boldsymbol{b}+\lambda \boldsymbol{b}_{p}\right\rfloor_{\mathrm{SOPOT}} .
$$

The vector $\boldsymbol{b}_{p}$ is a random vector with elements chosen in the range \pm 1 , and $\lambda$ is a user-defined variable used to control the size of the neighborhood to be searched. $\lfloor\cdot\rfloor_{\mathrm{SOPOT}}$ is the rounding operator that converts every element inside the input vector to its closest SOPOT value with a given value of $l$. The performance measure $\delta_{a}$ of the new coefficients is then calculated. The set that yields the minimum peak error $\delta_{a}$ under the given constraints of total number of terms and $l$ is recorded as the final solution. Since this is a random search algorithm, the longer the searching time, the higher the chance of finding the optimal solution. There are several advantages of this algorithm. First of all, with the computational power of personal computer (PC) nowadays, the time for obtaining high quality solutions is manageable. In fact, for the problem considered here, the overall design time takes less than 10 minutes to complete on a typical 


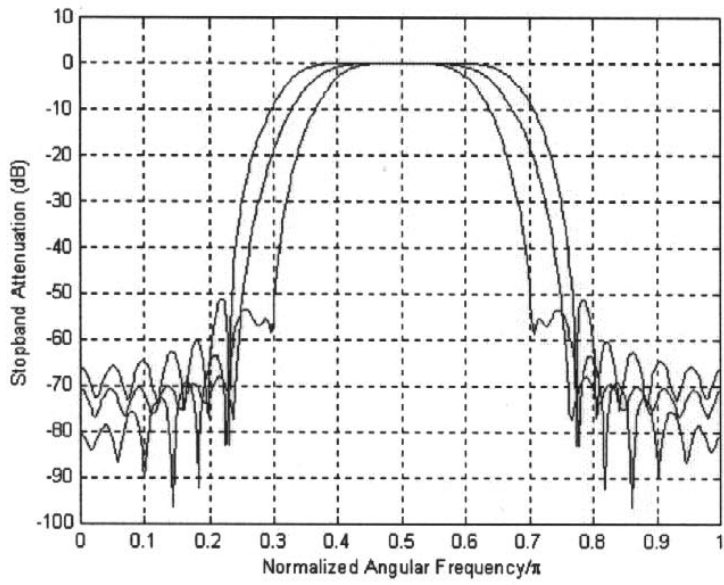

(a)

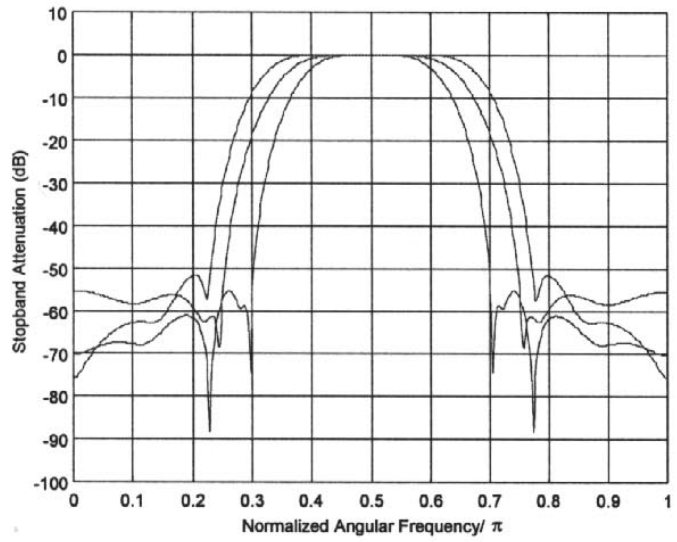

(c)

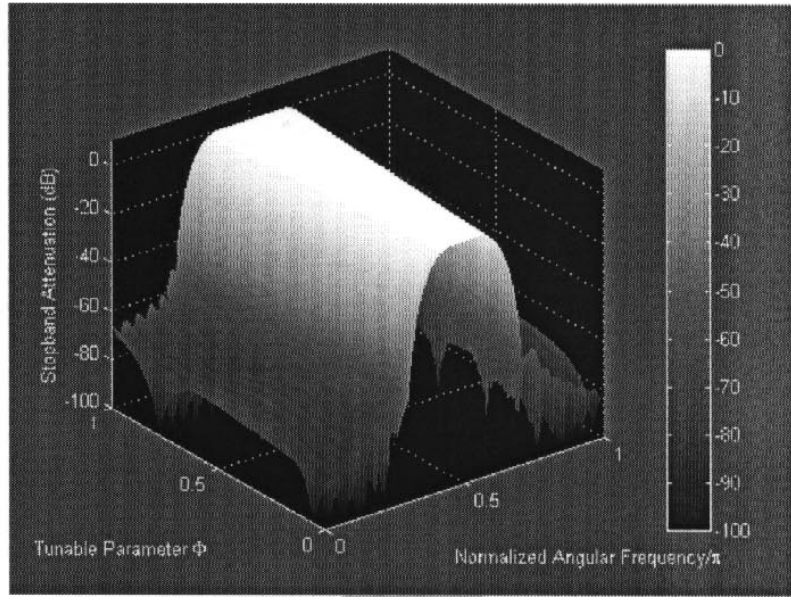

(b)

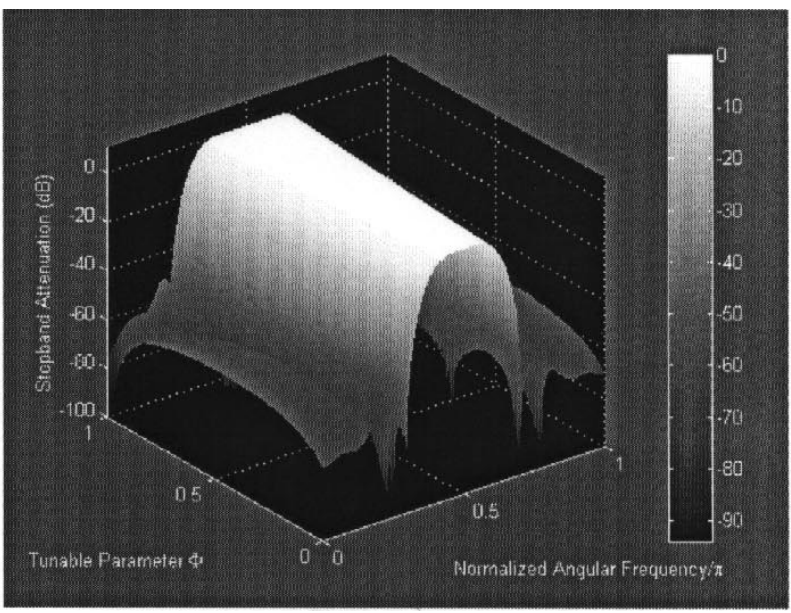

(d)

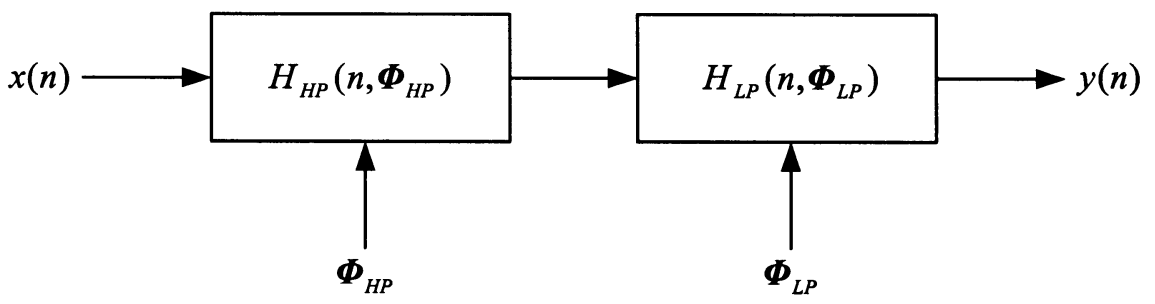

(e)

Fig. 7. Design results in Example 4. (a) Frequency response of the FIR Bandpass VDF at $\boldsymbol{\Phi}_{\mathrm{LP}}, \boldsymbol{\Phi}_{\mathrm{HP}}=0,0.5$, 1. (b) 3-D perspective plot of the frequency response of the FIR Bandpass VDF. (c) Frequency response of the IIR Bandpass VDF at $\boldsymbol{\Phi}_{\mathrm{LP}}, \boldsymbol{\Phi}_{\mathrm{HP}}=0,0.5,1.7$. (d) 3-D perspective plot of the frequency response of the IIR Bandpass VDF at $\boldsymbol{\Phi}=\boldsymbol{\Phi}_{\mathrm{LP}}=\boldsymbol{\Phi}_{\mathrm{HP}} \in[0.1]$. (e) Structure of the variable bandpass filter.

Pentium-400 PC using MATLAB ${ }^{1}$ 5.3, including both the design of SOPOT coefficients and the multiplier block. Secondly, it is applicable to problems with general objective functions probably with very complicated inequality constraints (such as round-off error [20] and power constraints). Moreover, a set of possible solutions representing different tradeoffs between computational complexity and performance will be generated during the search. The random search algorithm is similar in concept to the stimulated annealing algorithm. However, we have used the real-valued optimal solution as a starting point to reduce the searching time required.

${ }^{1}$ MATLAB is a registered trademark of The MathWorks, Natick, MA.
Detailed implementation of the FIR VDF will now be described. Referring to the general structure of the VDF in Fig. 1(a). To implement the subfilters $C_{m}(z)$ using the multiplier block, we can redraw them in transposed form as shown in Fig. 1(b). Now, we need to implement the multiplication of the input sample with a large number of constant coefficients in SOPOT form. These products can be efficiently implemented using a technique called the multiplier block. The basic idea of the multiplier block is to reduce the redundancies in multiplying a given input with a set of integer coefficients by removing any possible common subexpressions in their representations. Using the $\mathrm{MB}$, it is possible to reduce significantly the additions in implementing the multiplier-less subfilters leading to great 
hardware savings. The generation of the multiplier block in our case follows closely the work of [19]. From the design examples to be presented in the following section, it is observed that $2 / 3$ of the additions in implementing the SOPOT coefficient multiplications can be reduced by using the multiplier block.

\section{Design EXAMPLes}

\section{A. Example 1-Tunable Linear-Phase FIR and Approximately Linear-Phase IIR Low-Pass Filters}

In this example, tunable linear-phase FIR and IIR low-pass filters are designed using the proposed method. The transition bandwidth is fixed at $0.2 \pi$ and the passband edge is varied from $0.2 \pi$ to $0.2 \pi$. The FIR VDF has a subfilter length of 32 and an interpolation order of 5 using the polynomial basis function. The frequency response of the FIR VDF so obtained is shown in Fig. 3(a), (c), and (e). After model reduction, the lengths of the numerator and denominator polynomial are 17 . The frequency response, group delay and transient response of the IIR VDF are shown in Fig. 3(b), (d), (f), (g), and (h). It can be seen that the magnitude and phase responses of the IIR VDF are similar to that of FIR VDF. The parameters of the FIR and IIR VDFs are summarized in Table I. It can be observed that a modest amount of performance loss is given up in exchange for an approximate $40 \%$ reduction in multiplications. To examine the transient effect during parameter tuning, a sinusoid with a digital frequency of $0.4 \pi$ is applied to this IIR VDF. In the first 50 samples, $\boldsymbol{\Phi}$ is set to zero, and the cutoff frequency of the IIR VDF is around $0.2 \pi$. It can be seen that there is a transient response at the filter output when the data is first applied, which is an inherent characteristic of digital filters. This transient soon died down and the amplitude of the output is nearly zero. After that, the tuning parameter $\boldsymbol{\Phi}$ is increased by 0.25 every 50 samples, i.e., the passband is increased by $0.2 \pi$ successively. It can be seen that the amplitude of the VDF output increases at each block of 50 samples. This is because the input sinusoid starts to fall into the passband of the IIR VDF. However, except for the initial transient, no transients due to abrupt change in tuning parameter $\boldsymbol{\Phi}$ are observed. This is because the states of the IIR subfilters are not abruptly changed by the tuning parameters, unlike other VDF approaches based on direct tuning of the filter parameters. In summary, the IIR VDF approximates the FIR VDF well in terms of frequency response without any transients during tuning.

\section{B. Efficient Realization}

We now consider the efficient realization of the above FIR VDF. The random search algorithm mentioned in Section IV was used to determine the SOPOT coefficients of the subfilters. The average number of terms in each SOPOT coefficients was found to be 4.1, and the worst-case stopband attenuation is 46.1 dB. Fig. 4(a) shows the frequency responses of the VDF at different control parameters. It can be seen that the frequency response is approximately equal-ripple in the stopband over the entire tuning range. Table II summarizes the design results of these VDF low-pass filters. Another point worth mentioning is that the multiplier block (MB) is able to reduce the required number of adders to about one-third of its original value. Hence, the system complexity is dramatically reduced with
TABLE VI

DETAILED COMPARISON BETWEEN THE METHOD IN [13] AND THE PROPOSED LEAST SQUARES (LS) APPROACH $\omega_{s 1}(\boldsymbol{\Phi})$ AND $\omega_{p 1}(\boldsymbol{\Phi})$ REPRESENT THE LOWER STOPBAND AND PASSBAND EDGES OF THE BANDPASS VDF, RESPECTIVELY $\omega_{s 2}(\boldsymbol{\Phi})$ AND $\omega_{p 2}(\boldsymbol{\Phi})$ REPRESENT THE HIGHER STOPBAND AND PASSBAND EDGES OF THE BANDPASS VDF, RESPECTIVELY

\begin{tabular}{|c|c|c|}
\hline RESULTS & [13] & Proposed (LS) \\
\hline subfilter length $(N)$ & 51 & 51 \\
\hline number of subfilters $(L)$ & 6 & 6 \\
\hline spectral parameter $(\boldsymbol{\Phi})$ & $-0.1 \leq \boldsymbol{\Phi} \leq 0.1$ & $0 \leq \boldsymbol{\Phi} \leq 1$ \\
\hline stopband edge $1\left(\omega_{s 1}(\boldsymbol{\Phi})\right)$ & $(0.26+\Phi) \pi$ & $(0.36-0.2 \Phi) \pi$ \\
\hline passband edge $1\left(\omega_{p 1}(\Phi)\right)$ & $(0.32+\boldsymbol{\Phi}) \pi$ & $(0.42-0.2 \Phi) \pi$ \\
\hline passband edge $2\left(\omega_{p 2}(\boldsymbol{\Phi})\right)$ & $(0.64-\Phi) \pi$ & $(0.54+0.2 \Phi) \pi$ \\
\hline stop band edge $2\left(\omega_{s 2}(\Phi)\right)$ & $(0.70-\boldsymbol{\Phi}) \pi$ & $(0.60+0.2 \Phi) \pi$ \\
\hline \multirow{9}{*}{ maximum deviation } & 0.0240 for $\Phi=-0.1$ & 0.0456 for $\Phi=1$ \\
\hline & 0.0282 for $\Phi=-0.075$ & 0.0414 for $\Phi=0.875$ \\
\hline & 0.0356 for $\Phi=-0.05$ & 0.0442 for $\boldsymbol{\Phi}=0.75$ \\
\hline & 0.0263 for $\Phi=-0.025$ & 0.0211 for $\Phi=0.625$ \\
\hline & 0.0495 for $\Phi=0$ & 0.0258 for $\Phi=0.5$ \\
\hline & 0.0294 for $\boldsymbol{\Phi}=0.025$ & 0.0233 for $\boldsymbol{\Phi}=0.325$ \\
\hline & 0.0479 for $\Phi=0.05$ & 0.0446 for $\Phi=0.25$ \\
\hline & 0.0594 for $\boldsymbol{\Phi}=0.075$ & for $\Phi=0.125$ \\
\hline & 0.0438 for $\boldsymbol{\Phi}=0.1$ & 0.0571 for $\Phi=0$ \\
\hline $\begin{array}{l}\text { worst-case passband } \\
\text { deviation }\end{array}$ & N/A & 0.0587 \\
\hline $\begin{array}{l}\text { worst-case stopband } \\
\text { deviation }\end{array}$ & N/A & 0.0594 \\
\hline SOPOT wordlength & N/A & 12 bits \\
\hline adders before using $\mathrm{MB}$ * & N/A & 503 \\
\hline adders after using MB * & N/A & 142 \\
\hline
\end{tabular}

only slight or negligible degradation in performance. We have also designed a number of VDF using different orders of interpolation with the same filter specifications. Fig. 4(b) plots the worst-case stopband attenuation as a function of the order of the interpolation polynomial.

\section{Example 2 - Tunable Linear-Phase FIR and Approximate Linear-Phase IIR Low-Pass Filters Using Piecewise Polynomial}

In this example, a tunable linear-phase FIR VDF and an approximately linear-phase IIR VDF are designed. The target tuning range of the passband is from $0.2 \pi$ to $0.4 \pi$ and the transition bandwidth is fixed at $0.2 \pi$. Instead of using six branches of subfilters as in the previous example, we divide the tuning range into two intervals with three subfilters per interval. Each subfilter has 40 taps. The frequency response of the FIR VDF designed using the least squares method is shown in Fig. 5(a) and (c). The structure of the VDF is shown in Fig. 2(b). It can be seen that the transfer functions $H_{i}(z)$, $i=0, \ldots, 5$, are separated into two groups, and each group is associated with one tuning range. The outputs from one of the groups are connected to the matrix $\Psi^{-1}$ at any one time, through the multiplexer, and it is determined by the tuning parameter $\boldsymbol{\Phi}$. $\boldsymbol{\Phi}$ is limited between 0 and 2. For $\boldsymbol{\Phi} \in[0,1]$, $H_{i}(z), i=0,2,4$, are used and it supports the passband tuning range from $0.2 \pi$ to $0.3 \pi$. Whereas, for $\boldsymbol{\Phi} \in[1,2], H_{i}(z), i=$ $1,3,5$, are selected and its tuning range is from $0.3 \pi$ to $0.4 \pi$. The corresponding $\Psi^{-1}(m=3)$ is

$$
\Psi^{-1}=\left[\begin{array}{ccc}
1 & 0 & 0 \\
-3 & 4 & -1 \\
2 & -4 & 2
\end{array}\right] \text {. }
$$




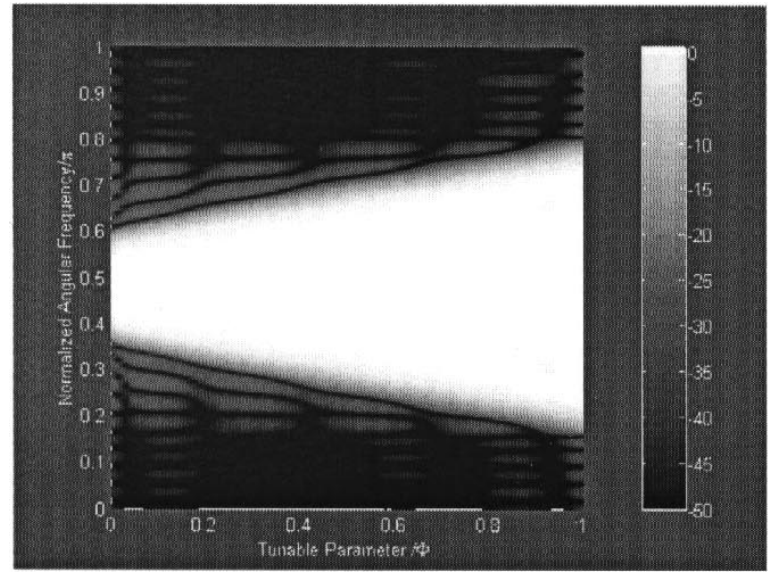

(a)

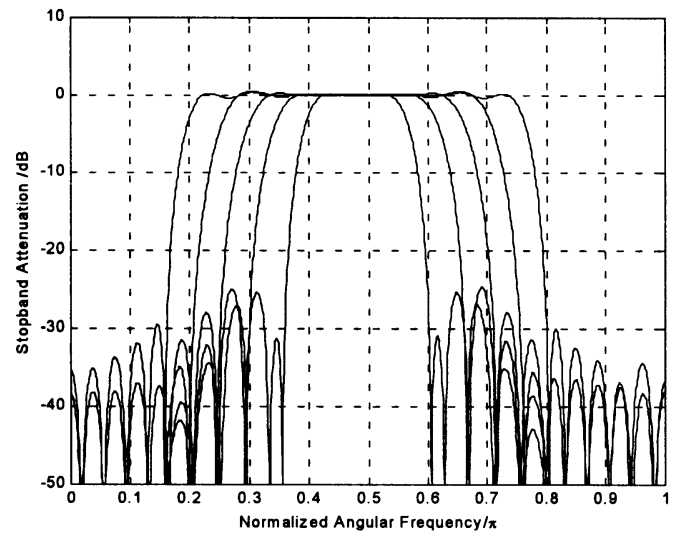

(c)

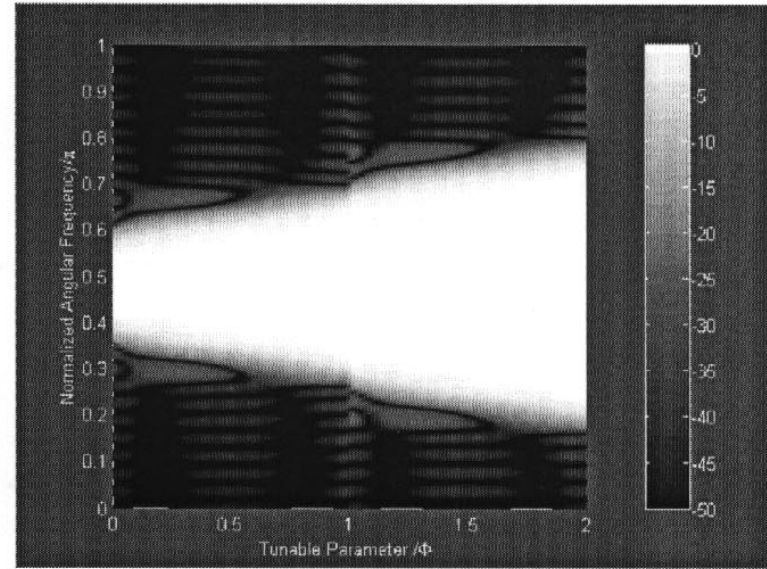

(b)

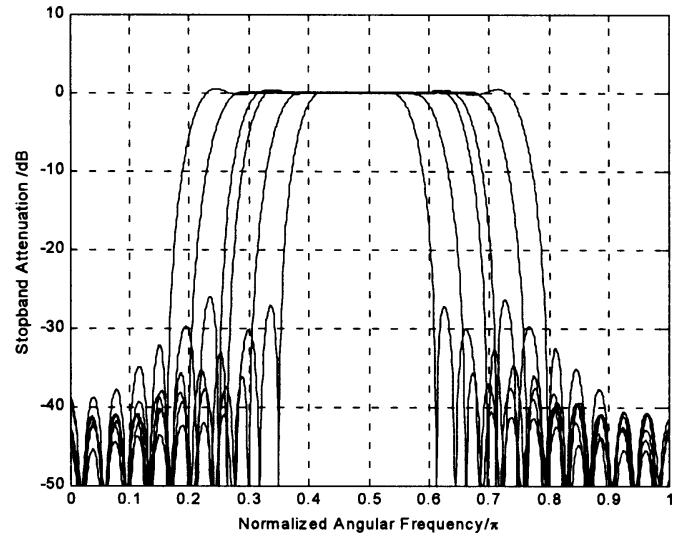

(d)

Fig. 8. Design results in Example 5: (a) Frequency responses of the digital bandpass VDF designed by using the LS approach. (b) Frequency responses of the digital bandpass VDF designed by using the piecewise polynomial approach. (c) Frequency responses of the digital bandpass VDF designed by using the LS approach $(\boldsymbol{\Phi}=0,0.25,0.5,0.75,1)$. (d) Frequency responses of the digital bandpass VDF designed by using with piecewise polynomial approach $\left(\boldsymbol{\Phi}_{1 \text { lower }}=\right.$ $\left.\Phi_{\text {upper }}=0,0.5,1\right)$.

The overall six-branch-FIR VDF is model reduced to obtain the required IIR VDF using the proposed method described in Section IV. The frequency response and group delay are plotted in Fig. 5(b), (d), and (e). Details comparison of the FIR and IIR VDF are summarized in Table III. Since the order of the IIR VDF is reduced to half and there is only one denominator for all the subfilters, the total number of multiplications is reduced approximately by $40 \%$ as compared with the FIR VDF. The frequency response of the IIR VDF is seen to be comparable to the original FIR VDF.

\section{Efficient Realization}

The random search algorithm was used to determine the SOPOT coefficients of the subfilters in the FIR VDF obtained above. The average number of terms in each SOPOT coefficients was found to be 3.8, and the worst-case stopband attenuation is $50 \mathrm{~dB}$. Table IV summarizes the design results of this VDF low-pass filter. Again, it can be seen that the multiplier block (MB) is able to reduce the required number of adders by around 65\%. Hence, the system complexity is dramatically reduced with only slight or negligible degradation in performance.

\section{E. Example 3 - Tunable Low-Delay FIR/IIR Low-Pass Filters Using Piecewise Polynomials}

In this example, tunable low-delay FIR/IIR low-pass filters using piecewise polynomials are designed. All the FIR VDF specifications are the same as the one in Example 2, except that the group delay of the FIR VDFs is now reduced. The group delay is reduced from 19.5 to 16.5 samples. The low-delay FIR VDF was first designed using the least squares design method. This low-delay FIR VDF was then model reduced to an IIR VDF using the ERA algorithm proposed in Section IV. The frequency responses and group delay are plotted in Fig. 6. Details comparison of the FIR and IIR VDF are summarized in Table V. From the figures, it can be seen that the frequency response of the IIR VDF closely resembles that of the FIR VDF and the number of multiplications is reduced by approximately $40 \%$. Due to space limitation, results for the multiplier-less realization is omitted.

\section{F. Example 4 - Bandpass Filter With Tunable Cutoff Frequencies}

In this example, we are going to design a bandpass filter with tunable cutoff frequency using the previous result on tunable low-pass filter. The basic idea is to cascade a tunable low-pass and a tunable high-pass filters together so that we can control its 
TABLE VII

Design Results Using THE PIECEWISE POLYNOMIAL AND LS APPROACHES $\omega_{s 1}(\boldsymbol{\Phi})$ AND $\omega_{p 1}(\boldsymbol{\Phi})$ REPRESENT THE LOWER STOPBAND AND PASSBAND EDGES OF THE BANDPASS VDF, RESPECTIVELY. $\omega_{s 2}(\boldsymbol{\Phi})$ AND $\omega_{p 2}(\boldsymbol{\Phi})$ REPRESENT THE HIGHER STOPBAND AND PASSBAND EDGES OF THE BANDPASS VDF, RESPECTIVELY

\begin{tabular}{|c|c|c|}
\hline RESULTS & VDF for the first section & VDF for the second section \\
\hline subfilter length $(N)$ & 51 & 51 \\
\hline number of subfilters $(L)$ & 3 & 3 \\
\hline spectral parameter $(\boldsymbol{\Phi})$ & $0 \leq \boldsymbol{\Phi}_{\text {upper }} \leq 1$ & $0 \leq \boldsymbol{\Phi}_{\text {lower }} \leq 1$ \\
\hline stopband edge $1\left(\omega_{s 1}(\boldsymbol{\Phi})\right)$ & $\left(0.26-0.1 \boldsymbol{\Phi}_{\text {upper }}\right) \pi$ & $\left(0.36-0.1 \boldsymbol{\Phi}_{\text {lower }}\right) \pi$ \\
\hline passband edge $1\left(\omega_{p 1}(\Phi)\right)$ & $\left(0.32-0.1 \Phi_{\text {upper }}\right) \pi$ & $\left(0.42-0.1 \boldsymbol{\Phi}_{\text {lower }}\right) \pi$ \\
\hline passband edge $2\left(\omega_{p 2}(\Phi)\right)$ & $\left(0.64+0.1 \Phi_{\text {upper }}\right) \pi$ & $\left(0.54+0.1 \boldsymbol{\Phi}_{\text {lower }}\right) \pi$ \\
\hline stop band edge $2\left(\omega_{s 2}(\Phi)\right)$ & $\left(0.70+0.1 \Phi_{\text {upper }}\right) \pi$ & $\left(0.60+0.1 \boldsymbol{\Phi}_{\text {lower }}\right) \pi$ \\
\hline \multirow{5}{*}{ maximum deviation } & 0.0505 for $\boldsymbol{\Phi}_{\text {upper }}=1$ & 0.0351 for $\boldsymbol{\Phi}_{\text {lower }}=1$ \\
\hline & 0.0478 for $\Phi_{\text {upper }}=0.75$ & 0.0483 for $\boldsymbol{\Phi}_{\text {lower }}=0.75$ \\
\hline & 0.0158 for $\boldsymbol{\Phi}_{\text {upper }}=0.5$ & 0.0158 for $\boldsymbol{\Phi}_{\text {lower }}=0.5$ \\
\hline & 0.0475 for $\Phi_{\text {upper }}=0.25$ & 0.0538 for $\boldsymbol{\Phi}_{\text {lower }}=0.25$ \\
\hline & 0.0495 for $\boldsymbol{\Phi}_{\text {upper }}=0$ & 0.0444 for $\boldsymbol{\Phi}_{\text {lower }}=0$ \\
\hline worst-case passband deviation & 0.0505 & 0.0485 \\
\hline worst-case stopband deviation & 0.0495 & 0.0548 \\
\hline SOPOT wordlength & 12 bits & 12 bits \\
\hline adders before using MB * & 128 & 133 \\
\hline adders after using MB * & 55 & 57 \\
\hline adders before using MB * & \multicolumn{2}{|c|}{219} \\
\hline adders after using MB * & \multicolumn{2}{|c|}{90} \\
\hline
\end{tabular}

* : using SOPOT coefficients.

cutoff frequencies individually. The proposed structure is illustrated in Fig. 7(e). It can be seen that the bandwidth of the filter can be controlled by two variables: $\boldsymbol{\Phi}_{\mathrm{LP}}$ and $\boldsymbol{\Phi}_{\mathrm{HP}}$, which control the cutoff frequencies of the low-pass filter $h_{\mathrm{LP}}(n)$ and the high-pass filter $h_{\mathrm{HP}}(n)$, respectively. It is possible to reduce the overall system delay by interpolating the impulse response of the filter by two-dimensional polynomial, but the overall complexity will increase dramatically with the square of the order used instead of linearly with the proposed cascade structure.

For simplicity, the tunable high-pass filter is obtained from the tunable low-pass filter $H_{\mathrm{LP}}\left(e^{j \omega}, \boldsymbol{\Phi}_{\mathrm{LP}}\right)$ using the following frequency transformation

$$
H_{\mathrm{HP}}\left(e^{j \omega}, \boldsymbol{\Phi}\right)=H_{\mathrm{LP}}\left(e^{j(\pi-\omega)}, \boldsymbol{\Phi}\right) .
$$

Hence, the overall VDF bandpass filter $H_{\mathrm{BP}}\left(e^{j \omega}, \mathbf{\Phi}_{\mathrm{LP}}, \boldsymbol{\Phi}_{\mathrm{HP}}\right)$ is given by

$$
H_{\mathrm{BP}}\left(e^{j \omega}, \boldsymbol{\Phi}_{\mathrm{LP}}, \boldsymbol{\Phi}_{\mathrm{HP}}\right)=H_{\mathrm{HP}}\left(e^{j \omega}, \boldsymbol{\Phi}_{\mathrm{HP}}\right) H_{\mathrm{LP}}\left(e^{j \omega}, \boldsymbol{\Phi}_{\mathrm{LP}}\right) .
$$

An interesting feature of this special structure is that the coefficients of $H_{\mathrm{LP}}\left(e^{j \omega}, \boldsymbol{\Phi}\right)$ and $H_{\mathrm{HP}}\left(e^{j \omega}, \boldsymbol{\Phi}\right)$ are identical except for appropriate sign changes. More precisely, if the low-pass $\mathrm{VDF}$ is given by $H_{\mathrm{LP}}\left(e^{j \omega}, \boldsymbol{\Phi}_{\mathrm{LP}}\right)=$ $\sum_{m=0}^{M-1}\left[\sum_{n=0}^{N-1} c_{n . m} e^{-j n \omega}\right] \boldsymbol{\Phi}_{\mathrm{LP}}^{m}$, then the high-pass VDF so obtained is given by $H_{\mathrm{HP}}\left(e^{j \omega}, \boldsymbol{\Phi}_{\mathrm{HP}}\right)=$ $\sum_{m=0}^{M-1}\left[\sum_{n=0}^{N-1} c_{n . m}(-1)^{n} e^{j n \omega}\right] \boldsymbol{\Phi}_{\mathrm{HP}}^{m}$. So, the filter coefficients of the low-pass VDF might be reused in the high-pass VDF, if the hardware is able to work at higher speed.

For simplicity, the low-pass VDF, $H_{\mathrm{LP}}\left(e^{j \omega}, \boldsymbol{\Phi}_{\mathrm{LP}}\right)$, is taken from Example 2. The frequency responses of the resulting bandpass FIR and IIR VDFs are shown in Fig. 7. From these figures, it can be seen that the IIR bandpass VDF approximates very well its FIR counterpart. Due to page limitation, results for their efficient realizations are omitted.

\section{G. Example 5}

The final example considered is the bandpass digital FIR VDF obtained using the outer product expansion in [13]. The center frequency of the VDF is kept constant at $0.5 \pi$, while its bandpass bandwidth is controlled by the spectral parameter $\boldsymbol{\Phi}$. The detailed specifications are shown in Table VI. Although we have used a different range for the parameter $\boldsymbol{\Phi}$ (between 0 and 1) from that of [13], the specifications for both VDFs are the same. The proposed least squares design and random search algorithms were used to obtain a VDF with SOPOT coefficient up to 12-bits wordlength. Its frequency response is plotted in Fig. 8(a) and (c). Table VI compares the result obtained in [13] with the proposed method. It can be seen that they are comparable to each other. On the other hand, the design of the proposed method is very simple because it requires the solution of a system of linear equation, instead of using nonlinear optimization in [13]. The number of adders saved in this bandpass FIR VDF with the use of MB is very significant. In fact, it saves nearly two-third of the adders in the SOPOT realization. Next, we compare the proposed piecewise polynomial approach with the conventional polynomial approach. The tuning range is divided into two nonoverlapping regions, and a bandpass FIR VDF is separately designed for each region. The details of these two VDFs are shown in Table VII and the frequency responses are plotted in Fig. 8(b) and (d). From these figures, it can be seen that the piecewise polynomial approach gives a better stopband attenuation than the conventional approach. The improvement will increase with wider tuning range. Through the use of MB, again, we can save approximately over one-half of the original adders.

\section{CONCLUSION}

A systematic method for the design and implementation of FIR and IIR VDFs is presented. A least squares (LS) approach for designing FIR VDFs by expressing the impulse response of the VDF as a linear combination of basis functions is first presented. The optimal LS solution can be obtained by solving a system of linear equations. By choosing the basis functions as piecewise polynomials, VDFs with larger tuning range than ordinary polynomial based approach can be obtained. The resulting VDFs can be efficiently implemented using the familiar Farrow structure. Making use of the FIR VDF so obtained, an ERA-based model reduction technique is proposed to approximate the FIR VDF by a stable IIR VDF with lower system order. The model reduction approach is computational simple, which only requires the computation of the singular value decomposition of a Hankel matrix. The IIR VDF obtained is guaranteed to be stable. In addition, the proposed IIR VDF does not suffer from undesirable transient response during parameter tuning found in other approaches based on direct tuning of filter parameters. For frequency selective VDFs, about $40 \%$ of the multiplications can be saved using the IIR VDF. The implementation of the proposed FIR VDF using sum-of-powers-of-two (SOPOT) 
coefficient and the multiplier block (MB) technique is also presented. Results show that about two-third of the additions in implementing the multiplication of the SOPOT coefficients can be saved using the multiplier block, which leads to significant savings in hardware complexity.

\section{ACKNOWLEDGMENT}

The authors would like to thank Dr. T. B. Deng for reprints of his papers on variable digital filters and useful discussion, which greatly improved the presentation of this paper.

\section{REFERENCES}

[1] F. M. Gardner, "Interpolation in digital modems. I. fundamentals," IEEE Trans. Commun., vol. 41, pp. 501-507, Mar. 1993.

[2] L. Erup, F. M. Gardner, and R. A. Harris, "Interpolation in digital modems. II. Implementation and performance," IEEE Trans. Commun. vol. 41, pp. 998-1008, June 1993.

[3] A. V. Oppenheim, W. F. G. Mecklenbrauker, and R. M. Mersereau, "Variable cutoff linear phase digital filters," IEEE Trans. Circuits Syst. II, vol. 23, pp. 199-203, Apr. 1976.

[4] P. Jarske, Y. Neuvo, and S. K. Mitra, "A simple approach to the design of linear phase FIR digital filters with variable characteristics," Signal Processing, vol. 14, no. 4, pp. 313-326, June 1988

[5] W. S. Lu and T. B. Deng, "An improved weighted least-squares design for variable fractional delay FIR filters," IEEE Trans. Circuits Syst. II, vol. 46, pp. 1035-1040, Aug. 1999.

[6] T. B. Deng, "Design of recursive 1-D variable filters with guaranteed stability," IEEE Trans. Circuits Syst. II, vol. 44, pp. 689-695, Sept. 1997.

[7] — " "Variable 2-D FIR digital filter design and parallel implementation," IEEE Trans. Circuits Syst. II, vol. 46, pp. 631-635, May 1999.

[8] C. K. S. Pun, S. C. Chan, and K. L. Ho, "Efficient 1D and circular symmetric 2D FIR filters with variable cutoff frequencies using the farrow structure and multiplier-block," in Proc. ISCAS, vol. 2, 2001, pp. $561-564$.

[9] C. W. Farrow, "A continuously variable digital delay element," in Proc. ISCAS, 1988, pp. 2641-2645.

[10] A. Tarczynski, G. D. Gain, E. Hermanowicz, and M. Rojewski, "WLS design of variable frequency response FIR filters," in Proc. ISCAS, vol. 4, 1997, pp. 2244-2247.

[11] A. G. Constantinides, "Spectral transformation for digital filters," in Proc. Inst. Elect. Eng., 1970, pp. 1585-1590.

[12] M. Makundi, T. I. Laakso, and V. Valimaki, "Efficient tunable IIR and allpass filter structures," Electron. Lett., vol. 37, pp. 344-345, Mar. 2001.

[13] T. B. Deng and T. Soma, "Variable digital filter design using the outer product expansion," IEE Proc. Vision, Image and Signal Processing, vol. 141, pp. 123-128, Apr. 1994.

[14] R. Zarour and M. M. Fahmy, "A design technique for variable digital filters," IEEE Trans. Circuits Syst. II, vol. 36, pp. 1473-1478, Nov. 1989.

[15] V. Valimaki, T. I. Laakso, and J. Mackenzie, "Eliminate of transients in time-varying allpass fractional delay filters with application to digital waveguide modeling," in Proc. Int. Computer Music Conf. (ICMC), Banff, Canada, Sept. 3-7, 1995, pp. 327-334.

[16] V. Valimaki and T. I. Laakso, "Suppression of transients in variable recursive digital filters with a novel and efficient cancellation method," IEEE Trans. Signal Processing, vol. 46, pp. 3408-3414, Dec. 1998.

[17] J. N. Juang and R. S. Pappa, "An eigensystem realization algorithm for modal parameter identification and model reduction," J. Guid. Control Dyn., vol. 8, no. 5, pp. 620-627, Sept.-Oct. 1985.

[18] J. N. Juang, J. E. Cooper, and J. R. Wright, "An eigensystem realization algorithm using data correlations (ERA/DC) for modal parameter identification," Control-Theory Adv. Technol., vol. 4, no. 1, pp. 5-14, 1988.

[19] A. G. Dempster and M. D. Macleod, "Use of minimum-adder multiplier blocks in FIR digital filters," IEEE Trans. Circuits Syst. II, vol. 42, pp. 569-577, Sept. 1995.

[20] C. K. S. Pun, S. C. Chan, and K. L. Ho, "Efficient design of a class of multiplier-less perfect reconstruction two-channel filter banks and wavelets with prescribed output accuracy," Proc. 11th IEEE Statistical Signal Processing Workshop, pp. 599-602, 2001.
[21] T. B. Deng, "Weighted least-squares method for designing arbitrarily variable 1-D FIR digital filters," Signal Processing, vol. 80, pp. 597-613, 2000.

[22] S. K. Mitra, Digital Signal Processing: A Computer-Based Approach, Singapore: McGraw-Hill, 1998.

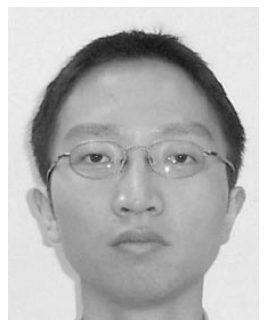

Carson K. S. Pun received the B.Eng. and M.Phil. degrees in electrical and electronic engineering from The University of Hong Kong, Hong Kong, in 2000 and 2002, respectively. $\mathrm{He}$ is currently pursuing the Ph.D. degree at the Department of Electrical and Computer Engineering in the University of California, San Diego.

His current research interests include the digital signal processing and its applications.

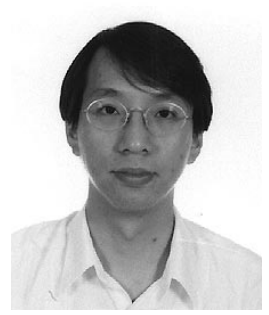

S. C. Chan received the B.Sc. (Eng) and Ph.D. degrees from The University of Hong Kong, Hong Kong, in 1986 and 1992, respectively.

In 1990, he joined City Polytechnic of Hong Kong, Kowloon, as an Assistant Lecturer and later as a University Lecturer. Since 1994, he has been with the Department of Electrical and Electronic Engineering, University of Hong Kong, where he is currently an Associate Professor. In 1998, he was a Visiting Researcher at Microsoft Corporation, Redmond, WA and in 1999 at Microsoft, Taiwan, R.O.C. His research interests include fast transform algorithms, filter design and realization, multirate signal processing, communications signal processing, and image-based rendering.

Dr. Chan is the Chairman of the IEEE Hong Kong Chapter of Signal Processing and a Member of the Digital Signal Processing Technical Committee of the IEEE Circuits and Systems Society.

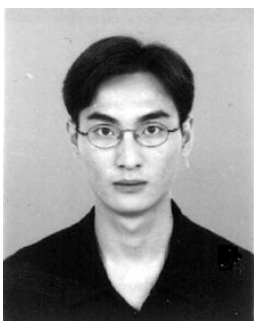

K. S. Yeung received the B.Eng. degree in electrical and electronic engineering from The University of Hong Kong, Hong Kong, in 2001. He is currently working toward the M.Phil. degree in the Department of Electrical and Electronic Engineering at the same university.

His main research interests include digital signal processing, multirate filter-banks and wavelets, digital filter design, and their efficient realization and applications.

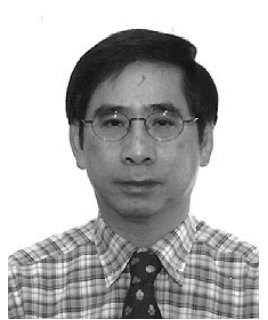

K. L. Ho (M'79) received the B.Sc. (Eng.) and the M.Phil. degrees in electrical engineering from The University of Hong Kong, Hong Kong, in 1971 and 1973, respectively, and the Ph.D degree from the University of London, London, U.K., in 1977.

In 1984, he joined the Department of Electrical and Electronic Engineering at The University of Hong Kong. His current research interests include wave propagation, signal processing and communications systems.

$\mathrm{He}$ is a Chartered Engineer of the Engineering Council, U.K., a Member of the Institute of Electrical Engineers (IEE), London, U.K., and the Hong Kong Institution of Engineers (HKIE). 